Nonlinear Processes in Geophysics, 12, 269-289, 2005

SRef-ID: $1607-7946 / \mathrm{npg} / 2005-12-269$

European Geosciences Union

(c) 2005 Author(s). This work is licensed

under a Creative Commons License.

\title{
The dynamics of electron and ion holes in a collisionless plasma
}

\author{
B. Eliasson and P. K. Shukla \\ Institut für Theoretische Physik IV, Fakultät für Physik und Astronomie, Ruhr-Universität Bochum, D-44780 Bochum, \\ Germany
}

Received: 2 November 2004 - Revised: 26 January 2005 - Accepted: 27 January 2005 - Published: 11 February 2005

Part of Special Issue "Nonlinear plasma waves-solitons, periodic waves and oscillations"

\begin{abstract}
We present a review of recent analytical and numerical studies of the dynamics of electron and ion holes in a collisionless plasma. The new results are based on the class of analytic solutions which were found by Schamel more than three decades ago, and which here work as initial conditions to numerical simulations of the dynamics of ion and electron holes and their interaction with radiation and the background plasma. Our analytic and numerical studies reveal that ion holes in an electron-ion plasma can trap Langmuir waves, due the local electron density depletion associated with the negative ion hole potential. Since the scalelength of the ion holes are on a relatively small Debye scale, the trapped Langmuir waves are Landau damped. We also find that colliding ion holes accelerate electron streams by the negative ion hole potentials, and that these streams of electrons excite Langmuir waves due to a streaming instability. In our Vlasov simulation of two colliding ion holes, the holes survive the collision and after the collision, the electron distribution becomes flat-topped between the two ion holes due to the ion hole potentials which work as potential barriers for low-energy electrons. Our study of the dynamics between electron holes and the ion background reveals that standing electron holes can be accelerated by the selfcreated ion cavity owing to the positive electron hole potential. Vlasov simulations show that electron holes are repelled by ion density minima and attracted by ion density maxima. We also present an extension of Schamel's theory to relativistically hot plasmas, where the relativistic mass increase of the accelerated electrons have a dramatic effect on the electron hole, with an increase in the electron hole potential and in the width of the electron hole. A study of the interaction between electromagnetic waves with relativistic electron holes shows that electromagnetic waves can be both linearly and nonlinearly trapped in the electron hole, which widens further due to the relativistic mass increase and ponderomotive force in the oscillating electromagnetic field. The results of
\end{abstract}

Correspondence to: B. Eliasson

(bengt@tp4.rub.de) our simulations could be helpful to understand the nonlinear dynamics of electron and ion holes in space and laboratory plasmas.

\section{Introduction}

About a quarter century ago, Schamel (1971, 1972, 1979, 1986; Bujarbarua and Schamel, 1981) presented a theory for ion and electron holes, where a vortex distribution is assigned for the trapped particles, and where the integration over the trapped and untrapped particle species in velocity space gives the particle number density as a function of the electrostatic potential. The potential is then calculated self-consistently from Poisson's equation. This model has been used in theoretical analyses of the existence criteria for the ion and electron holes (Bujarbarua and Schamel, 1981), and in the analysis of stability of phase space holes (Schamel, 1982, 1986).

Numerical and theoretical studies of the interaction between electron and ion holes have been performed by several authors. Newman et al. (2001) in their numerical experiments studied the dynamics and instability of twodimensional phase-space tubes, and Daldorff et al. (2001) investigated the formation and dynamics of ion holes in thee dimensions. Krasovsky et al. (1999) showed theoretically and by computer simulations that that electron holes perform inelastic collisions, and Vetoulis (2001) studied the radiation generation due to bounce resonances in electron holes. Guio et al. $(2003,2004)$ have studied numerically the dynamics of phase space vortices in a collisionless plasma as well as the generation of phase space structures by an obstacle in a streaming plasma. Saeki and Genma (1998) studied the disruption of electron holes in an electron-ion plasma. The asymptotic nonlinear saturation of electrostatic waves has been treated both theoretically (Medvedev et al., 1998; Lancelotti and Dorning, 1998) and numerically (Manfredi, 1997). It has also been pointed out that plasma waves can be undamped due to particle trapping effects in waves with arbitrarily small amplitudes (Holloway and Dorning, 1991; 
Schamel, 2000). Theoretical investigations of trapped particle effects in magnetized plasmas shows that trapped ions influence strongly ion cyclotron waves (Abbasi et al., 1999).

In laboratory experiments, the formation and dynamics of solitary electron holes (Saeki et al., 1979; Petraconi and Macel, 2003) and ion holes (Pécseli et al, 1981, 1984, 1987; Nakamura et al., 1999) as well as accelerated ion holes (Franck et al., 2001) have been observed. Observations of broad electrostatic noise (BEN) by the GEOTAIL (Matsumoto et al., 1994) and FAST spacecrafts (Ergun et al., 1998a, b, 2001) in the auroral acceleration regions have revealed that BEN is connected to solitary electron BGK modes/electron holes. Recent observations by the WIND satellite in the Earth's bow shock also reveal localized structures with bipolar electric fields typical for the electron BGK modes/holes (Bale, 1998, 2002; Hoshino, 2003). The geomagnetic field-aligned bipolar electric field pulses associated with electron phase space holes have also been observed by the Polar and Cluster spacecrafts in the magnetosheath and at the Earth's magnetopause (Cattel et al., 2002; Dombeck et al., 2001; Pickett et al., 2002, 2004; Drake et al., 2003), while signatures of ion holes have been observed by the Fast Auroral Snapshot (FAST) spacecraft (McFadden et al., 2003) and by the Viking satellite (Boström, et al., 1988).

The interaction between low-frequency ion-acoustic waves with high-frequency Langmuir turbulence and electromagnetic waves was described more than three decades ago by Hasegawa (1970), Karpman (1971, 1975a, b) and Zakharov (1972), in which high-frequency photons and plasmons interact nonlinearly with low-frequency ion-acoustic waves via the ponderomotive force arising due to the spatial gradient of the high-frequency wave intensity. This nonlinear interaction is typically described by the two-fluid and Poisson-Maxwell equations, and the governing equations admit the localization of photon and plasmon wavepackets, leading to the formation of envelope light and Langmuir wave solitons (Rudakov, 1972; Varma and Rao, 1980; Rao and Varma, 1982; Schamel, 1977). The latter are composed of electron/ion density depression which traps photon and Langmuir wave envelops. Schamel and Maslov (1999) studied theoretically the contraction of Langmuir waves trapped in small-amplitude electron holes. Califano and Lontano (1998) and Wang et al. (1997) studied numerically by Vlasov simulations the dynamics of finite-amplitude high-frequency Langmuir waves in an one-dimensional electron-ion plasma, where wave collapse and heating of the electrons have been observed. In two and three dimensions, one encounters photon self-focusing and Langmuir wave collapse (Zakharov, 1972; Shapiro and Shevchenko 1984; Goldman, 1984). The formation of cavitons has been observed in the ionosphere (Wong, 1974) as well as in several laboratory experiments (Ikezi, 1976; Intrator, 1984; Wong, 1975). Yan'kov (1979) studied the response of kinetic untrapped ions in the Langmuir envelope soliton theory, and predicted the formation of sub ion thermal small-amplitude negative potential wells in plasmas. On the other hand, Mokhov and Chukbar (1984) found a Langmuir envelope soliton accompanied with small- amplitude negative potential well created by localized Langmuir wave electric fields in a quasi-neutral plasma with nonisothermal ions whose temperature is much smaller than the electron thermal temperature.

Relativistic effects play a very important role in highenergy laser-plasma experiments (Shukla, 1986; Montgomery, 2001), in plasma based electron and photon accelerators (Bingham, 2003; Bingham et al., 2004; Mendonça, 2001), in supernova remnants and in gamma ray bursts (Piran, 1999), where electrons can be accelerated to relativistic energies by strong electrostatic fields. For short laser pulse intensities exceeding $10^{19} \mathrm{~W} / \mathrm{cm}^{2}$, electrons in the laser beam oscillate relativistically. Interactions between intense short laser pulses and background plasma give rise to a number of nonlinear effects (Mendonça, 2001; Shukla et al., 1986; Bingham et al., 2003; Bingham, 2004) associated with relativistic electron mass increase in the electromagnetic fields and the plasma density modification due to relativistic radiation ponderomotive force. In the past, several authors presented theoretical (Kaw et al., 1992; Esirkepov et al., 1998) and particle-in-cell simulation (Esirkepov et al., 1998; Bulanov et al., 1999; Farina and Bulanov, 2001; Naumova et al., 2001) studies of intense electromagnetic envelope solitons in a cold plasma where the plasma slow response to the electromagnetic waves is modeled by the electron continuity and relativistic momentum equations, supplemented by Poisson's equation. Experimental observations (Borghesi et al., 2002) have shown bubble-like structures in proton images of laser-produced plasmas, which are interpreted as remnants of electromagnetic envelope solitons.

In this paper, we present a review of recent theoretical and numerical results of the interaction between ion and electron holes with the background plasma. Specifically, we find that colliding ion holes may lead to non-Maxwellian electron distributions due to the acceleration of electrons in the negative ion hole potential, which works as a barrier for the negatively charged electrons (Eliasson and Shukla, 2004a). Streams of electrons are accelerated by the colliding ion holes, and these electron streams can excite high-frequency Langmuir waves due to a streaming instability. On the other hand, Langmuir waves can be trapped in the density cavity of the ion holes, as described by a nonlinear Schrödinger equation for the Langmuir waves (Shukla and Eliasson, 2003). Numerical studies show that the trapped Langmuir waves are Landau damped due to the relatively small Debye-scale of the ion holes (Eliasson and Shukla, 2004a). We have also studied the fully nonlinear interaction between electron holes and oxygen ions by means of a Vlasov simulations (Eliasson and Shukla, 2004b), where it is found that the large-amplitude electron hole potential accelerates the ions locally and that the self-created ion density cavity accelerates the electron hole, which propagates away from the ion cavity with a constant speed close to half the electron thermal speed. Finally, we have extended the idea of Schamel to relativistic plasma, where the trapped and free particles are given by solutions of the relativistic Vlasov equation. We have studied theoretically and numerically the properties of relativistic electron 
holes, and their interaction with relativistically high amplitude electromagnetic waves.

The article is organized as follows. In Sect. 2, we review the general theory of ion and electron holes based on Schamel's solution of the quasi-stationary Vlasov-Poisson system. The dynamics of ion holes in plasmas and their interaction with high-frequency waves and kinetic electrons is discussed in Sect. 3. In Sect. 4, we study the dynamics of electron holes in a plasma with mobile ions. An extension of Schamel's theory is presented in Sect. 5, where relativistic electron holes and their interactions with intense electromagnetic radiation are studied theoretically and numerically. Finally, conclusions are drawn in Sect. 6.

\section{General theory of ion and electron holes}

The dynamics of electrons and ions in an unmagnetized, collisionless plasma is governed by the Vlasov-Poisson system

$\frac{\partial f_{j}}{\partial t}+v \frac{\partial f_{j}}{\partial x}-\frac{q_{j}}{m_{j}} \frac{\partial \varphi}{\partial x} \frac{\partial f_{j}}{\partial v}=0$,

and

$\frac{\partial^{2} \varphi}{\partial x^{2}}=-4 \pi \sum_{j} q_{j} n_{j}$,

where the number density is

$n_{j}=\int_{-\infty}^{\infty} f_{j} d v$.

Here $f_{j}$ is the distribution function of the particle species $j$ ( $j$ equals $e$ for electrons and $i$ for ions), $m_{j}$ is the mass, $q_{e}=-e, q_{i}=e, e$ is the magnitude of the electron charge, $E=-\partial \varphi / \partial x$ is the electric field, and $\varphi$ is the electrostatic potential. In Fig. 1, we have solved numerically the Vlasov equation for the electrons, while the ions have been assumed to have a constant density, $n_{i}=n_{0}$. Here, the initial condition is a large-amplitude, density-modulated wave, which is released at $t=0 \omega_{p e}^{-1}$, and which then evolves in time, where $\omega_{p e}=\left(4 \pi n_{0} e^{2} / m_{e}\right)^{1 / 2}$ is the electron plasma frequency and $n_{0}$ is the equilibrium electron number density. We see that at $t=7 \omega_{p e}^{-1}$, some of the electrons have been accelerated by the self-consistent electrostatic field and have formed streams in velocity space. At $t=70 \omega_{p e}^{-1}$, the streams of electrons have mixed further in a complex fashion. Two electron holes have been created at $v \approx \pm 3 V_{T e}$, associated with a depletion of the electron distribution function and a population of trapped electrons moving with the electron holes. Here, $V_{T e}=\left(T_{e} / m_{e}\right)^{1 / 2}$ is the electron thermal speed and $T_{e}$ is the electron temperature.

In order to describe ion and electron holes mathematically, we look for quasi-stationary structures moving with velocity $v_{0}$. In this case, we make the ansatz $f_{j}=f_{j}(\xi, v)$ and $\varphi=$ $\varphi(\xi)$, where $\xi=x-u_{0} t$, which transforms the system of Eqs. (1) and (2) to a new set of equations

$\left(-u_{0}+v\right) \frac{\partial f_{j}}{\partial \xi}-\frac{q_{j}}{m_{j}} \frac{\partial \varphi}{\partial x} \frac{\partial f_{j}}{\partial v}=0$,

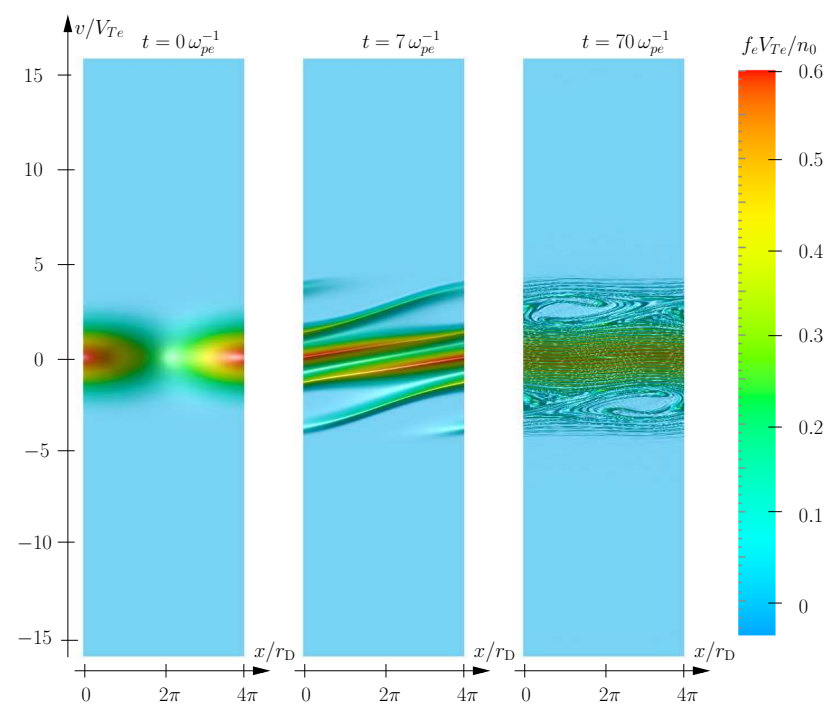

Fig. 1. The electron distribution function at $t=0 \omega_{p e}^{-1}$ (left panel), $t=7 \omega_{p e}^{-1}$ (middle panel) and $t=70 \omega_{p e}^{-1}$ (lower panel). The initial distribution function at $t=0 \omega_{p e}^{-1}$ is $f=n_{0}\left(m_{e} / 2 \pi T_{e}\right)^{1 / 2}[1+$ $\left.0.5 \cos \left(0.5 x / r_{D}\right)\right] \exp \left(-m_{e} v^{2} / 2 T_{e}\right)$.

and

$\frac{d^{2} \varphi}{d \xi^{2}}=-4 \pi e \int_{-\infty}^{\infty}\left(f_{i}-f_{e}\right) d v$.

Equation 4 can be integrated along its particle trajectories, so that the distribution functions $f_{j}$ are functions of the particle energy

$\mathcal{E}_{i}=\frac{m_{i}\left(v-u_{0}\right)^{2}}{2}+e \varphi$

and

$\mathcal{E}_{e}=\frac{m_{e}\left(v-u_{0}\right)^{2}}{2}-e \varphi$

for ions and electrons, respectively. Here, $\mathcal{E}_{j}>0$ corresponds to untrapped particles, while $\mathcal{E}_{j}<0$ corresponds to trapped particles, when $\varphi \rightarrow 0$ at $|\xi|=\infty$.

Electron holes are characterized by a localized positive potential, in which a population of the negatively charged electrons can be trapped. A special class of electron hole solutions can be found by prescribing the distribution function for the trapped and free electrons (Schamel 1971, 1972),

$$
f_{e}= \begin{cases}a_{e} \exp \left\{-\frac{1}{T_{e}}\left[ \pm \mathcal{E}_{e}^{1 / 2}+\left(\frac{m_{e} u_{0}^{2}}{2}\right)^{1 / 2}\right]^{2}\right\}, & \mathcal{E}_{e}>0 \\ a_{e} \exp \left[-\frac{1}{T_{e}}\left(\beta \mathcal{E}_{e}+\frac{m_{e} u_{0}^{2}}{2}\right)\right], & \mathcal{E}_{e}<0\end{cases}
$$

where $a_{e}=n_{0}\left(m_{e} / 2 \pi T_{e}\right)^{1 / 2}$, and $\beta$ is the trapping parameter describing the "temperatures" of the trapped electrons. Negative values of $\beta$, which we are interested in here, leads to a vortex distributions of the trapped electrons, where the 


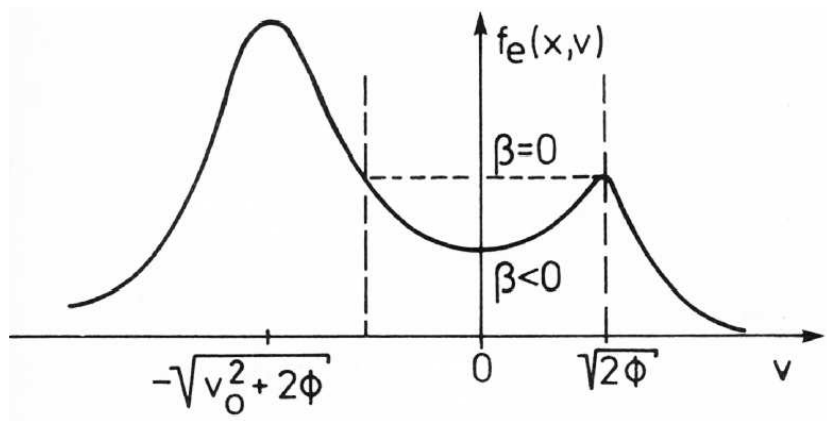

Fig. 2. The electron distribution function in velocity space (from Schamel, 1986). In a frame moving with speed of the the electron hole, electrons with a velocity $|v|<\sqrt{2 \phi}$ are trapped. Negative $\beta$ corresponds to an excavated vortex distribution, while $\beta=0$ corresponds to a flat (constant) distribution. The velocity $v$ is scaled by $\sqrt{T_{e} / m_{e}}$, and the potential $\phi$ by $T_{e} / e$.

distribution function is excavated locally. This is illustrated in Figs. 2 and 3 (taken from Schamel, 1986), where electrons with negative energies are trapped in an excavated region of the electron distribution function. In the study of electron holes which are moving much faster than the ion sound speed $\left(T_{e} / m_{i}\right)^{1 / 2}$, one can assume that the ions form a constant neutralizing background, $n_{i}=n_{0}$. We discuss a deviation from this treatment for the case of standing electron hole in Sect. 4, where the ion dynamics becomes important and where the electron holes are accelerated by the self-created ion density. Integration of the electron distribution function over velocity space gives the electron density as a function of the potential, $n_{e}(\varphi)$, which inserted into the Poisson equation gives an equation for the self-consistent potential (Schamel, 1971, 2000).

Ion holes are characterized by a localized negative potential, which can trap a population of the ions. Similarly as for the electron holes, a special class of solutions can be found by prescribing the ion distribution function for free $\left(\mathcal{E}_{i}>0\right)$ and trapped $\left(\mathcal{E}_{i}<0\right)$ ions as (Bujarbarua and Schamel, 1981)

$f_{i}= \begin{cases}a_{i} \exp \left\{-\frac{1}{T_{i}}\left[ \pm \mathcal{E}_{i}^{1 / 2}+\left(\frac{m_{i} u_{0}^{2}}{2}\right)^{1 / 2}\right]^{2}\right\}, & \mathcal{E}_{i}>0, \\ a_{i} \exp \left[-\frac{1}{T_{i}}\left(\alpha \mathcal{E}_{i}+\frac{m_{i} u_{0}^{2}}{2}\right)\right], & \mathcal{E}_{i}<0,\end{cases}$

where $a_{i}=n_{0}\left(m_{i} / 2 \pi T_{i}\right)^{1 / 2}, T_{i}$ is the ion temperature and $\alpha$ is the trapping parameter which describes the "temperature" of the trapped ions. We are interested in negative values on $\alpha$, giving an excavated vortex distribution of the ions. In the study of ion holes, the electrons are usually assumed to be Maxwell-Boltzmann distributed,

$f_{e}=n_{0}\left(\frac{m_{e}}{2 \pi T_{e}}\right)^{1 / 2} \exp \left(\frac{e \varphi}{T_{e}}-\frac{m_{e} v^{2}}{2 T_{e}}\right)$,

which simplifies the mathematics somewhat compared to using an exact solution of the Vlasov equation for electrons. We will see, however, in the following section that

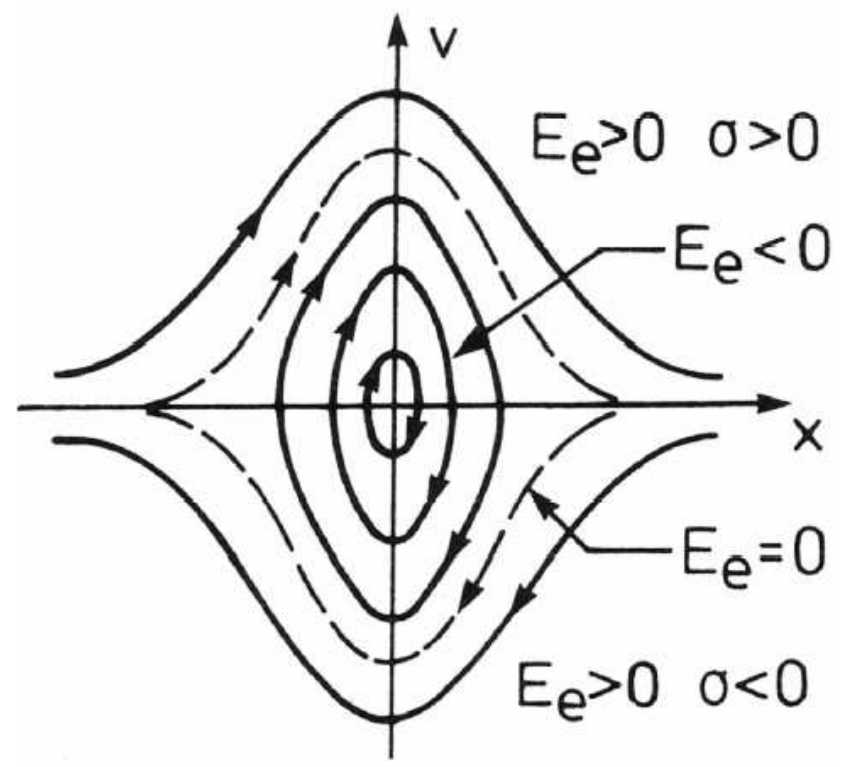

Fig. 3. Particle trajectories in the vicinity of an electron hole (from Schamel, 1986), for different normalized (by $T_{e}$ ) energies $E_{e}$. Trapped electrons have negative energies $\left(E_{e}<0\right)$, while free electrons have positive energies $\left(E_{e}>0\right)$. Here, $\sigma$ denotes the sign of the velocities of the free electrons, in a frame moving with the speed of the electron hole.

the dynamical interaction between ion holes may change the electron distribution so that it deviates strongly from the Maxwellian distribution. By integrating the distributions for the free and trapped ions and the electrons, both the ion and electron densities can be expressed as functions of the potential, $n_{i}(\varphi)$ and $n_{e}(\varphi)$, respectively. Inserting the particle number densities into the Poisson equation, one obtains an equation for the self-consistent potential of the ion hole, which may be solved analytically by means the Sagdeevpotential method (Schamel, 1971, 2000), or numerically as a nonlinear boundary value problem (Eliasson and Shukla, 2004a).

\section{The dynamics of ion holes in plasmas}

In the present section, we will study some kinetic effects associated with the interaction between ion holes and the background electron-ion plasma. Ion holes are associated with a negative electrostatic potential, which repels the electrons. Therefore, low-energy electrons can be trapped between two ion holes, and during collisions between the ion holes, the low-energy electrons are accelerated to high energies by the ion hole potential, forming electron streams escaping the two colliding ion holes. Streams of electrons can excite highfrequency Langmuir waves due to a streaming instability, and thus the processes on the slower ion timescale gives rise to high-frequency waves on an electron timescale. On the other hand, ion holes are characterized by a depletion of the electrons, which gives the possibility that high-frequency Lang- 

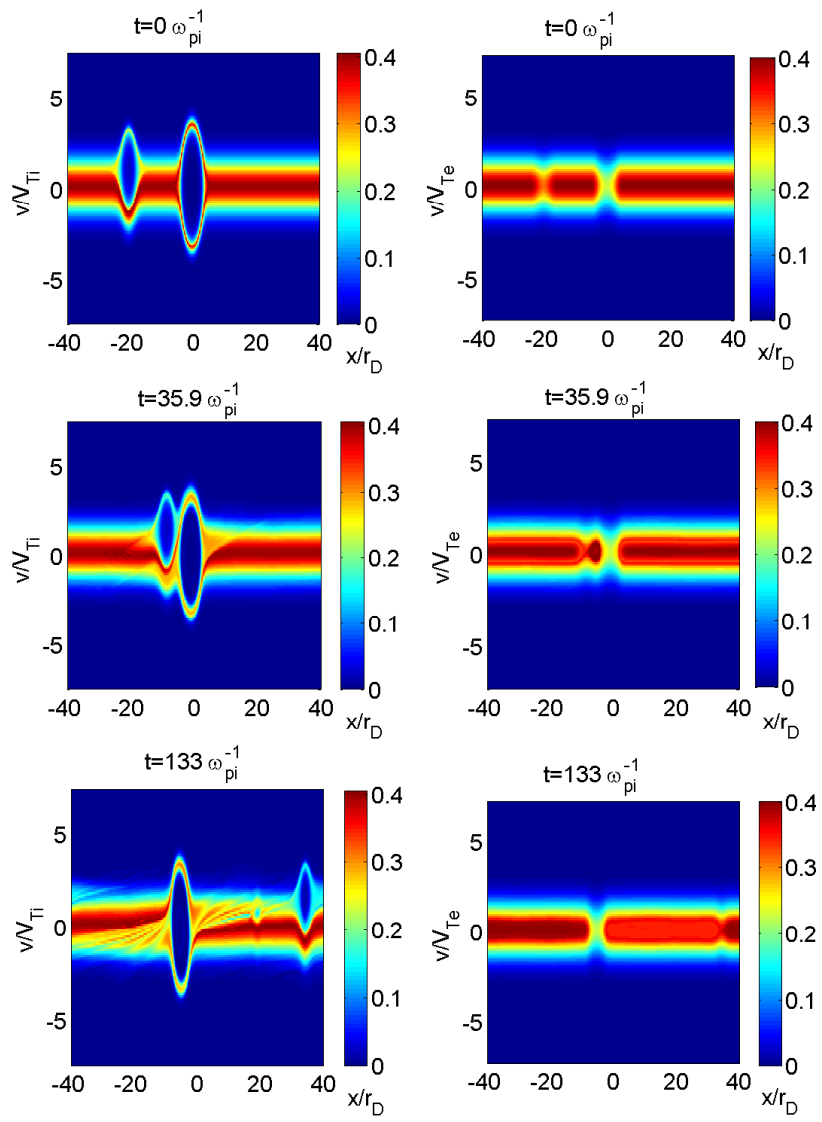

Fig. 4. The scaled ion distribution function $f_{i} V_{T i} / n_{0}$ (left panels) and electron distribution function $f_{e} V_{T e} / n_{0}$ (right panels) of two colliding ion holes, before the collision at times $t=0 \omega_{p i}^{-1}$ (upper panel) and $t=35.9 \omega_{p i}^{-1}$ (middle panel), and after the collision at $t=133 \omega_{p i}^{-1}$ (lower panel). The color bar goes from dark blue (small values) to dark red (large values).

muir waves can be trapped in the ion hole. Clearly, if the Langmuir waves have a large amplitude, there will be a modification of the ion hole due to the ponderomotive force acting on the electrons, which are repelled in the direction of decreasing Langmuir electric field amplitudes. These electrons then change the ion hole potential, as described via the Poisson equation. Thus, the interaction between the ion holes with the background plasma gives rise to a multitude of complex phenomena on different timescales, which we will explore theoretically and numerically in the present section.

In order to investigate the time-dependent dynamics of ion holes as well as kinetic effects for electrons, we employ a newly developed code (Eliasson, 2001), which numerically solves the Vlasov-Poisson system of Eqs. (1) and (2). The initial conditions for $f_{i}$ is taken as the Schamel distribution (Schamel, 1986) for the free and trapped ions, which in the rest frame of the bulk plasma is given by Eq. (9). We use (Shukla and Eliasson, 2003) $m_{i} / m_{e}=1836, \alpha=-1.0$, and $T_{e} / T_{i}=10$. The initial condition for the electron distribution function is taken to be the Maxwell-Boltzmann dis-
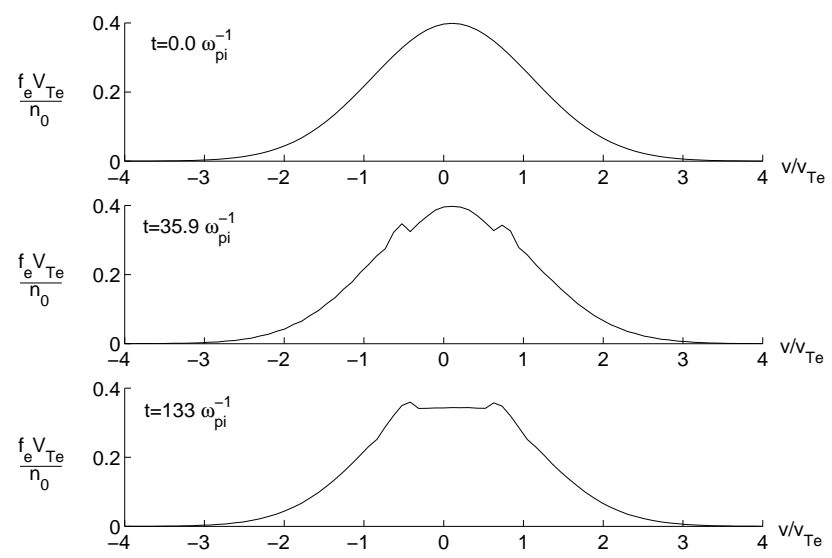

Fig. 5. The electron velocity distribution at $x=8 r_{D}$, for $t=$ $0 \omega_{p i}^{-1}$ (upper panel), $t=35.9 \omega_{p i}^{-1}$ (middle panel) and $t=133 \omega_{p i}^{-1}$ (lower panel).

tribution, given by Eq. (10). The potential $\varphi$ for the initial conditions (9) and (10) is obtained by inserting them into Eq. (5), which is then integrated (Bujarbarua and Schamel, 1981; Shukla and Eliasson, 2003) to obtain the potential $\varphi$ as a function of $x$; in practice the integration of Eq. (5) is performed numerically. The potential thus obtained is then inserted into Eqs. (9) and (10) to obtain the initial conditions for the ion and electron distribution functions.

First, we investigate the process of two colliding ion holes, as shown in Figs. 4-6. Here, $r_{D}=V_{T e} / \omega_{p e}=$ $\left(T_{e} / 4 \pi n_{0} e^{2}\right)^{1 / 2}$ is the electron Debye length. For the initial conditions, we considered that the ion holes initially are well separated in space, and that the interaction between the ion holes is weak, so that the solutions for the single ion holes can be matched in space to form an initial condition for the case with two ion holes. Figure 4 displays the features of the ion and electron distribution functions for two colliding ion holes, where initially (upper panels) the left ion hole propagates with the speed $u_{0}=0.9 V_{T i}$, where $V_{T i}=\left(T_{i} / m_{i}\right)^{1 / 2}$ is the ion thermal speed, and the right ion hole is standing. The ion and electron distribution functions associated with the ion holes are shown before collision at times $t=0 \omega_{p i}^{-1}$ (upper panels) and $t=35.9 \omega_{p i}^{-1}$ (middle panels), and after collision at time $t=133 \omega_{p i}^{-1}$ (lower panels). The left panels of Fig. 4 exhibit that the two ion holes undergo collisions without being destroyed, and as can be seen in the right panels of Fig. 4, the electrons have a strongly nonMaxwellian flat top distribution in the region between the ion holes after that the collision has taken place. We have plotted the electron velocity distribution function against $v / V_{T e}$ at $x=8.0 r_{D}$ in Fig. 5. We see that the initial Maxwellian distribution (the upper panel) changes to a distribution with beams at $v \approx \pm 0.6 V_{T e}$ (the middle panel) slightly before collision, and to a flat-top distribution with two maxima after collision (the lower panel). The reason for the creation of the flat-topped velocity distribution is that the two ion holes are associated with negative electrostatic potentials, and the elec- 

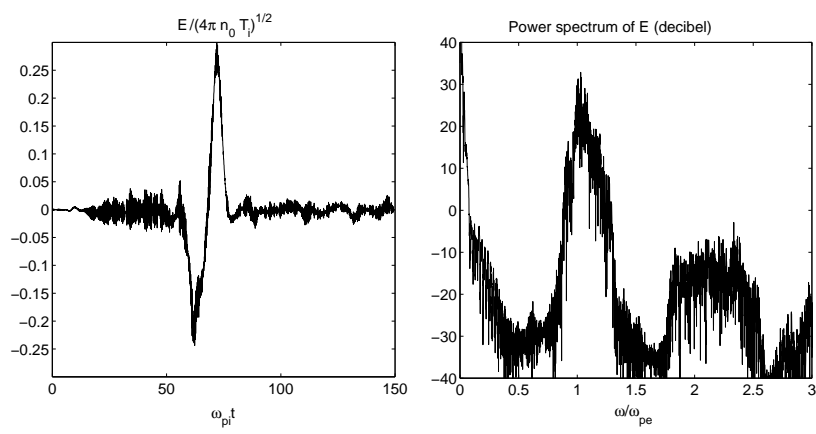

Fig. 6. The electric field at $x=8.0 r_{D}$ as a function of $\omega_{p i} t$ (left panel) and the power spectrum of the electric field as a function of $\omega / \omega_{p e}$ (right panel).

trons entering the region between the ion holes after collision must have a large enough kinetic energy to cross the potential barriers that are set up by the ion holes. The flattening of the low-velocity region then occurs probably due to repeated reflections and slowing down of the trapped electrons against the two moving potential barriers. On the other hand, we also observe that before collision, the low-energy population of electrons are compressed between the ion holes (see the middle right panel of Fig. 4) and released during collision; these electrons are then accelerated to form beams of electrons (see the middle panel of Fig. 5) which escape the ion holes. The energy of the electron beams are then released in a beam-plasma instability triggering high-frequency plasma oscillations, as illustrated in Fig. 6 (the left panel) where the electric field at $x=8.0 r_{D}$ is plotted as a function of $\omega_{p i} t$. High-frequency plasma oscillations are beginning to be excited at time $t \approx 10 \omega_{p i}^{-1}$. The large-amplitude bipolar electric fields of the ion hole appear as it crosses $x=8.0 r_{D}$ at time $t \approx 50-80 \omega_{p i}^{-1}$. By examining the frequency spectrum of the electric field (the right panel), we notice that the highfrequency oscillations have broad frequency spectrum which is up-shifted compared to the electron plasma frequency. We also have a slightly "downshifted" frequency band around $\omega / \omega_{p e}=0.9$, which we may be the frequencies of Langmuir waves trapped in the ion holes, as described below.

The numerical solutions of the Vlasov-Poisson system were performed by means of a Fourier transform method (Eliasson, 2001). We used 500 intervals in $x$ space with the domain $-40 \leq x / r_{D} \leq 40$ with periodic boundary conditions. We used 300 intervals in velocity space. The electron and ion speed intervals were in the ranges $-15.7 \leq v / V_{T e} \leq$ 15.7 and $-0.118 \leq v / V_{T e} \leq 0.118$, respectively. The timestep $\Delta t \approx 0.013 \omega_{p e}^{-1}$ was adapted dynamically to maintain numerical stability.

\subsection{Trapping of Langmuir waves in ion holes}

Ion holes are associated with a local depletion of both ions and electrons, the latter due to the negative ion hole potential. Clearly, there is a possibility that waves could be trapped in this plasma density cavity if the surrounding plasma is overdense so that the waves are prevented from escaping the cavity. A similar scenario was investigated theoretically by Schamel and Maslov (1999) for the trapping of Langmuir waves in small-amplitude electron holes. In the present section, we investigate the trapping of Langmuir waves in ion holes, while in Sect. 5 we consider the trapping of electromagnetic waves in electron holes.

We here consider an unmagnetized electron-ion plasma in the presence of Langmuir waves and large-amplitude ion holes. At equilibrium, we have $n_{e 0}=n_{i 0}=n_{0}$, where $n_{j 0}$ is the unperturbed number density of the particle species $j$ ( $j$ equals $e$ for electrons and $i$ for ions). The linear Langmuir wave frequency is $\omega_{0}=\left(\omega_{p e}^{2}+3 k_{0}^{2} V_{T e}^{2}\right)^{1 / 2}$, where $k_{0}$ is the wavenumber. Large-amplitude Langmuir waves interacting nonlinearly with ion holes generate Langmuir wave envelope whose electric field $E$ evolves slowly (in comparison with the electron plasma wave period) according to a nonlinear Schrödinger equation

$2 i \omega_{p e}\left(\frac{\partial}{\partial t}+v_{g} \frac{\partial}{\partial x}\right) E+3 V_{T e}^{2} \frac{\partial^{2} E}{\partial x^{2}}+\omega_{p e}^{2}\left(1-\frac{n_{e}}{n_{0}}\right) E=0$,

where $v_{g}=3 k_{0} V_{T e}^{2} / \omega_{p e}$ is the group velocity of the Langmuir waves. We note that Eq. (11) has been derived by combining the electron continuity and momentum equations as well as by using Poisson's equation with fixed ions, and by retaining the arbitrary large electron number density variation $n_{e}$ associated with the ion holes in the presence of the Langmuir wave ponderomotive force. Assuming that the phase speed of ion holes is much smaller than the electron thermal speed, we readily obtain from the inertialess electron equation of motion the electron number density in the presence of the ponderomotive force of the Langmuir waves. The result is

$n_{e}=n_{0} \exp \left[\tau\left(\tilde{\phi}-W^{2}\right)\right]$,

where $\tau=T_{i} / T_{e}, W=|E| /\left(16 \pi n_{0} T_{i}\right)^{1 / 2}$ is the scaled Langmuir wave envelope, and $\tilde{\phi}=e \varphi / T_{i}$ is the scaled electrostatic potential of the ion hole. We note that the $W^{2}$-term in Eq. (12) comes from the averaging of the nonlinear term $m_{e} v_{h e} \partial v_{h e} / \partial x$ over the Langmuir wave period $2 \pi / \omega_{p e}$, where $v_{h e} \approx-e E_{h} / m_{e} \omega_{p e}$ is the high-frequency electron quiver velocity in the Langmuir wave electric field $E_{h}=(1 / 2) E \exp \left(i k_{0} x-i w_{0} t\right)+$ complex conjugate.

If the potential has a maximum $\tilde{\phi}_{\max }>0$, then there exist, in general, trapped ions where $\tilde{\phi}<\tilde{\phi}_{\max }$, while at the point where $\tilde{\phi}=\tilde{\phi}_{\max }$ there are no trapped ions. Similar to Schamel (1971), we chose at this point a Maxwellian distribution for the free ions. The ion distribution function associated with the ion holes can then be obtained by solving the ion Vlasov equation for free and trapped ions, which have speeds larger and smaller than $\left[2\left(\tilde{\phi}_{\max }-\tilde{\phi}\right)\right]^{1 / 2}$, respectively. The electric potential will turn out to be essentially negative, with only a small-amplitude positive maximum $\tilde{\phi}_{\max }$ compared to the large-amplitude negative potential well with a minimum at $\tilde{\phi}_{\min } \equiv-\psi$. Thus, the potential is restricted by 
$-\psi \leq \tilde{\phi} \leq \tilde{\phi}_{\max }$, where $\psi$ plays the role of the amplitude. Integrating the sum of the free and trapped ion distribution functions over velocity space, we obtain the ion number density (Schamel, 1971)

$n_{i}=n_{0} b \exp \left(-\frac{M_{i}^{2}}{2}\right)\left\{I\left(\tilde{\phi}_{\max }-\tilde{\phi}\right)\right.$
$\left.+\kappa\left(\frac{M_{i}^{2}}{2}, \tilde{\phi}_{\max }-\tilde{\phi}\right)+\frac{2}{\sqrt{\pi|\alpha|}} W_{D}\left[\sqrt{\alpha\left(\tilde{\phi}-\tilde{\phi}_{\max }\right)}\right]\right\}$,

where $M_{i}=u_{0} / V_{T i}$ is the Mach number, and $u_{0}$ is the ion hole speed. The normalization constant

$b=\exp \left(\frac{M_{i}^{2}}{2}\right)\left\{I\left(\tilde{\phi}_{\max }\right)\right.$

$\left.+\kappa\left(\frac{M_{i}^{2}}{2}, \tilde{\phi}_{\max }\right)+\frac{2}{\sqrt{\pi|\alpha|}} W_{D}\left[\sqrt{-\alpha \tilde{\phi}_{\max }}\right]\right\}^{-1}$,

is chosen so that when $\tilde{\phi}=0$, the total density of ions is $n_{0}$. The special functions are defined as (Bujarbarua and Schamel, 1981)

$I(\Phi)=\exp (\Phi)[1-\operatorname{erf}(\sqrt{\Phi})]$,

$\kappa(X, \Phi)=\frac{2}{\sqrt{\pi}} \int_{0}^{\pi / 2} \sqrt{X} \cos \theta \exp \left(-\Phi \tan ^{2} \theta\right.$

$\left.+X \cos ^{2} \theta\right) \operatorname{erf}(\sqrt{X} \cos \theta) d \theta$,

and the Dawson integral

$W_{D}(Z)=\exp \left(-Z^{2}\right) \int_{0}^{Z} \exp \left(t^{2}\right) d t$

We are here mostly interested in negative values of $\alpha$; however, for positive $\alpha$, we use (Abramowitz and Stegun, 1972) $W_{D}(i Z)=i(\sqrt{\pi} / 2) \exp \left(Z^{2}\right) \operatorname{erf}(Z)($ where $i=\sqrt{-1})$ and replace the term $(2 / \sqrt{\pi|\alpha|}) W_{D}\left[\sqrt{\alpha\left(\tilde{\phi}-\tilde{\phi}_{\max }\right)}\right]$ in Eq. (13) by $(1 / \sqrt{\alpha}) \exp \left[-\alpha\left(\tilde{\phi}-\tilde{\phi}_{\max }\right)\right] \operatorname{erf}\left[\sqrt{-\alpha\left(\tilde{\phi}-\tilde{\phi}_{\max }\right)}\right]$; we note especially that $M_{i}=0, \alpha=1$ leads to a Boltzmann distribution $n_{i}=n_{0} \exp (-\tilde{\phi})$ for the ion number density. The Langmuir wave ponderomotive force acting on the ions is weaker by the electron to ion mass ratio in comparison with the one acting on the electrons, and therefore it is ignored in Eq. (13). The electron ponderomotive force is transmitted to ions via the ambipolar potential $\tilde{\phi}$, which is determined from Poisson's equation

$\tau r_{D}^{2} \frac{\partial^{2} \tilde{\phi}}{\partial x^{2}}=N_{e}-N_{i}$,

where $N_{e}=n_{e} / n_{0}$ and $N_{i}=n_{i} / n_{0}$.

We are interested in quasi-steady state solutions of Eqs. (11)-(16), which are fully nonlinear. We insert $E(x, t)=W(\xi) \exp \{i[K x-\Theta t]\}$ and $\tilde{\phi}=\tilde{\phi}(\xi)$ where $\xi=\left(x-u_{0} t\right) / r_{D}$, and where $W(\xi), K$ and $\Theta$ are assumed to be real, into Eqs. (11)-(16) and obtain a coupled set of nonlinear equations

$3 \frac{\partial^{2} W}{\partial \xi^{2}}-(\lambda-1) W-W \exp \left[\tau\left(\tilde{\phi}-W^{2}\right)\right]=0$, and

$$
\begin{aligned}
& \tau \frac{\partial^{2} \tilde{\phi}}{\partial \xi^{2}}-\exp \left[\tau\left(\tilde{\phi}-W^{2}\right)\right]+b \exp \left(-\frac{M_{i}^{2}}{2}\right) \times \\
& \left\{I\left(\tilde{\phi}_{\max }-\tilde{\phi}\right)+K\left[\frac{M_{i}^{2}}{2}, \tilde{\phi}_{\max }-\tilde{\phi}\right]\right. \\
& +\frac{2}{\sqrt{\pi|\alpha|}} W_{D}\left[\sqrt{\alpha\left(\tilde{\phi}-\tilde{\phi}_{\max }\right)}\right\}=0,
\end{aligned}
$$

where $\lambda=-2 \omega_{p e}^{-1} \Theta-3 k^{2} r_{D}^{2}\left(1-u_{0}^{2} / v_{g}^{2}\right)=-2 \omega_{p e}^{-1} \Theta-$ $3 k^{2} r_{D}^{2}+u_{0}^{2} / 3 V_{T e}^{2}$ represents a nonlinear frequency shift. The system of Eqs. (17) and (19) admits the first integral in the form of a Hamiltonian

$$
\begin{aligned}
& H\left(W, \tilde{\phi} ; M_{i}, \tau, \alpha, \lambda\right)=3\left(\frac{\partial W}{\partial \xi}\right)^{2}-\frac{\tau}{2}\left(\frac{\partial \tilde{\phi}}{\partial \xi}\right)^{2} \\
& -(\lambda-1) W^{2}+\frac{1}{\tau}\left\{\exp \left[\tau\left(\tilde{\phi}-W^{2}\right)\right]-1\right\} \\
& +b \exp \left(-\frac{M_{i}^{2}}{2}\right)\left[P\left(\tilde{\phi}_{\max }-\tilde{\phi}, \alpha\right)\right. \\
& \left.+h\left(\frac{M_{i}^{2}}{2}, 0, \tilde{\phi}_{\max }-\tilde{\phi}\right)-1\right]-H_{0}=0
\end{aligned}
$$

where in the unperturbed state $(|\xi|=\infty)$ we have used the boundary conditions $W=0, \tilde{\phi}=0, \partial W / \partial \xi=0, \partial \tilde{\phi} / \partial \xi=$ 0 . The constant

$$
H_{0}=b \exp \left(-\frac{M_{i}^{2}}{2}\right)\left[P\left(\tilde{\phi}_{\max }, \alpha\right)+h\left(\frac{M_{i}^{2}}{2}, 0, \tilde{\phi}_{\max }\right)-1\right]
$$

is chosen so that $H=0$ at $|\xi|=\infty$. The auxiliary functions are defined as

$$
\begin{aligned}
& P(\Phi, \alpha)=I(\Phi)+2 \sqrt{\frac{\Phi}{\pi}}\left(1-\alpha^{-1}\right) \\
& +\frac{2}{\alpha \sqrt{\pi|y|}} W_{D}(\sqrt{-\Phi \alpha}),
\end{aligned}
$$

and

$h(X, a, b)=\int_{a}^{b} \kappa(X, \Phi) d \Phi$.

Because we are interested in symmetric solutions defined by $W(\xi)=W(-\xi)$ and $\tilde{\phi}(\xi)=\tilde{\phi}(-\xi)$, the appropriate boundary conditions at $\xi=0$ are $W=W_{0}, \tilde{\phi}=-\psi$, $\partial W / \partial \xi=0$, and $\partial \tilde{\phi} / \partial \xi=0$. Hence, from Eq. (19) we have

$$
\begin{aligned}
& (\lambda-1) W_{0}^{2}-\frac{1}{\tau}\left\{\exp \left[\tau\left(-\psi-W_{0}^{2}\right)\right]-1\right\} \\
& -b \exp \left(-\frac{M_{i}^{2}}{2}\right)\left[P\left(\tilde{\phi}_{\max }+\psi, \alpha\right)\right. \\
& \left.+h\left(\frac{M_{i}^{2}}{2}, 0, \tilde{\phi}_{\max }+\psi\right)-1\right]-H_{0}=0,
\end{aligned}
$$

which shows how the maximum values of $W_{0}$ and $\psi$ are related to $M_{i}, \tilde{\phi}_{\max }$ and $\lambda$ for given values of $\tau$ and $\alpha$. A 

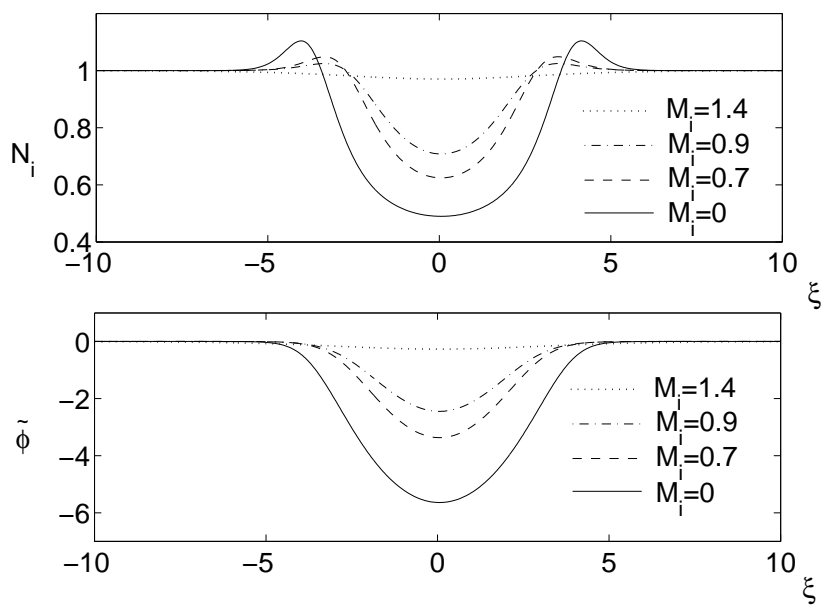

Fig. 7. The ion density (upper panel) and the potential (lower panel) of ion holes without Langmuir waves $(W=0)$ for different Mach numbers $M_{i}$, with $\tau=0.1$ and $\alpha=-1.0$.

practical application of the Hamiltonian (19) is to check the correctness of any numerical scheme used to solve Eqs. (17) and (19), while Eq. (23) depicts the parameter regimes for the existence of trapped Langmuir waves in ion holes.

In the absence of the Langmuir waves, ion holes are governed by the energy integral (Sagdeev, 1966)

$\frac{1}{2}\left(\frac{\partial \tilde{\phi}}{\partial \xi}\right)^{2}+V_{S}\left(\tilde{\phi} ; M_{i}, \tau, \alpha\right)=0$,

where the Sagdeev potential for our purposes with $\tilde{\phi}_{\max }=0$ is (Bujarbarua and Schamel, 1981)

$$
\begin{aligned}
& V_{S}\left(\tilde{\phi} ; M_{i}, \tau, \alpha\right)=-\frac{1}{\tau}\left\{\frac{1}{\tau}[\exp (\tau \tilde{\phi})-1]\right. \\
& \left.+\exp \left(-\frac{M_{i}^{2}}{2}\right)\left[P(-\tilde{\phi}, \alpha)+h\left(\frac{M_{i}^{2}}{2}, 0,-\tilde{\phi}\right)-1\right]\right\} .
\end{aligned}
$$

Equation (23), which is obtained from Eq. (19) in the limit of vanishing Langmuir wave electric fields, determines the profile of ion holes. The latter exist provided that $V_{S}\left(\tilde{\phi} ; M_{i}, \tau, \alpha\right)$ is negative for $\tilde{\phi}$ between zero and $-\psi$. The condition $V_{S}\left(-\psi ; M_{i}, \tau, \alpha\right)=0$ gives a relation between $\psi$ and $M_{i}$ for given values of $\alpha$ and $\tau$. It turns out that ion holes without large-amplitude Langmuir waves have only negative potentials, as pre-assumed earlier.

We have carried out numerical studies of the equations governing ion holes with and without Langmuir waves for $\tau=0.1$ and $\alpha=-1.0$. First, we consider small-amplitude Langmuir waves which are not strong enough to modify the ion hole, but which can be linearly trapped in the electron density well of the hole. Accordingly, for $W^{2} \ll 1 \mathrm{Eq}$. (17) turns into a linear eigenvalue problem of the form

$3 \frac{d^{2} W}{d \xi^{2}}+[1-\exp (\tau \tilde{\phi})-\lambda] W=0$, with the eigenvalue $\lambda$ and the corresponding eigenfunction $W$, and where $\tilde{\phi}$ is obtained by assuming $W=0$ in the solution of Eq. (19); in the upper and lower panels of Fig. 7, we display the ion density profiles and the associated ambipolar potentials for different sets of parameters, where the obtained negative potentials are used in the eigenvalue problem (25).

The eigenvalue problem (25) has a continuous spectrum for $\lambda<0$, associated with non-localized, propagating Langmuir wave envelopes $W$, and a point spectrum for $\lambda>0$, corresponding to localized, trapped Langmuir wave envelopes. We have investigated numerically the cases corresponding to four different Mach numbers displayed in Fig. 7, and found the corresponding positive eigenvalues listed in the left columns of Table 1 , where each eigenvalue $\lambda$ is associated to a bell-shaped eigenfunction $W$. Using $k_{0}=0$ in the definition of $\lambda$, we obtain the oscillation frequency of the trapped Langmuir waves as $\omega / \omega_{p e}=1+\Theta=$ $1-\lambda / 2+\left(u_{0} / V_{T e}\right)^{2} / 6=1-\lambda / 2+M_{i}^{2} \tau m_{e} / 6 m_{i}$. Only one positive eigenvalue was found for each case, admitting only the ground states for the Langmuir waves to be linearly trapped.

Next, we examine the influence of finite-amplitude Langmuir waves on the ion hole, in which the fully nonlinear system of Eqs. (17) and (18) has to be solved numerically, and where we use the same parameters as in the linear treatment above. The numerical solutions reveal that the ion hole deepened and widened, admitting the eigenvalue $\lambda$ to become larger. We have investigated the special case with a nonlinear shift of 0.1 of $\lambda$, listed in the column with nonlinear eigenvalues in Table 1, and have found solutions for all cases except for $M_{i}=1.4$; the numerical solutions are depicted in Fig. 8. Comparing with Fig. 7, we see that the presence of trapped finite-amplitude Langmuir waves makes the ion density depletion both deeper and wider, and the same holds for the ambipolar potential well. The deepening of the ambipolar negative potential well is a feature closely related to the strongly non-isothermal trapped ion distribution function. For this case, the electrostatic potential had small-amplitude maxima $\tilde{\phi}_{\max }$ of the order $\approx 10^{-3}$ on each side of the ion hole, and this maximum of the potential increased with increasing $M_{i}$.

Physically, the broadening of the ion hole and the enhancement of negative ambipolar potential occur because the ponderomotive force of the Langmuir waves locally expels electrons, which pull ions along to maintain the local charge neutrality. The deficit of the ions in plasmas, in turn, produces more negative potential within the ion hole that is now widened and enlarged to trap the localized Langmuir wave electric field envelope.

In order to investigate the conditions for the existence of ion holes in the presence of strong Langmuir fields, we numerically solved Eq. (22) for $\psi$ as a function of $M_{i}$; see Fig. 9. We used the same parameters $\tau=0.1$ and $\alpha=-1.0$ as above. Here, we assumed the Langmuir field to be given as an external parameter (say $W_{0}=0.8$ ) and with a nonlinear shift that approximately follows the relation $\lambda\left(M_{i}\right)=$ $0.3-0.14 M_{i}$ obtained from the fourth column of Table 1 . 
Table 1. Theoretical eigenvalues $\lambda$ and normalized frequencies $\omega / \omega_{p e}$ for trapped Langmuir waves inside ion holes propagating with different Mach numbers $M_{i}$. Theoretical linear values are compared with numerical values obtained from direct Vlasov simulation (left and middle columns). Eigenvalues (and frequencies) used in the the nonlinear theoretical treatment of large-amplitude trapped Langmuir wave envelopes are shown in the right columns.

\begin{tabular}{|c|c|c|c|c|c|}
\hline $\begin{array}{l}\text { Mach } \\
\text { number }\end{array}$ & \multicolumn{2}{|c|}{$\begin{array}{l}\text { Linear eigenvalues } \\
\text { and frequencies }\end{array}$} & $\begin{array}{l}\text { Frequencies from } \\
\text { Vlasov simulation }\end{array}$ & \multicolumn{2}{|c|}{$\begin{array}{l}\text { Nonlinear eigenvalues } \\
\text { and frequencies }\end{array}$} \\
\hline$M_{i}$ & $\lambda$ & $\omega / \omega_{p e}$ & $\omega / \omega_{p e}=\left(\omega_{r}+\mathrm{i} \gamma\right) / \omega_{p e}$ & $\lambda$ & $\omega / \omega_{p e}$ \\
\hline 1.4 & 0.0013 & 0.999 & & - & - \\
\hline 0.9 & 0.0463 & 0.977 & $0.98-0.00 \mathrm{i}$ & 0.1463 & 0.927 \\
\hline 0.7 & 0.0772 & 0.961 & & 0.1772 & 0.911 \\
\hline 0.0 & 0.1906 & 0.905 & $0.93-0.03 \mathrm{i}$ & 0.2906 & 0.855 \\
\hline
\end{tabular}
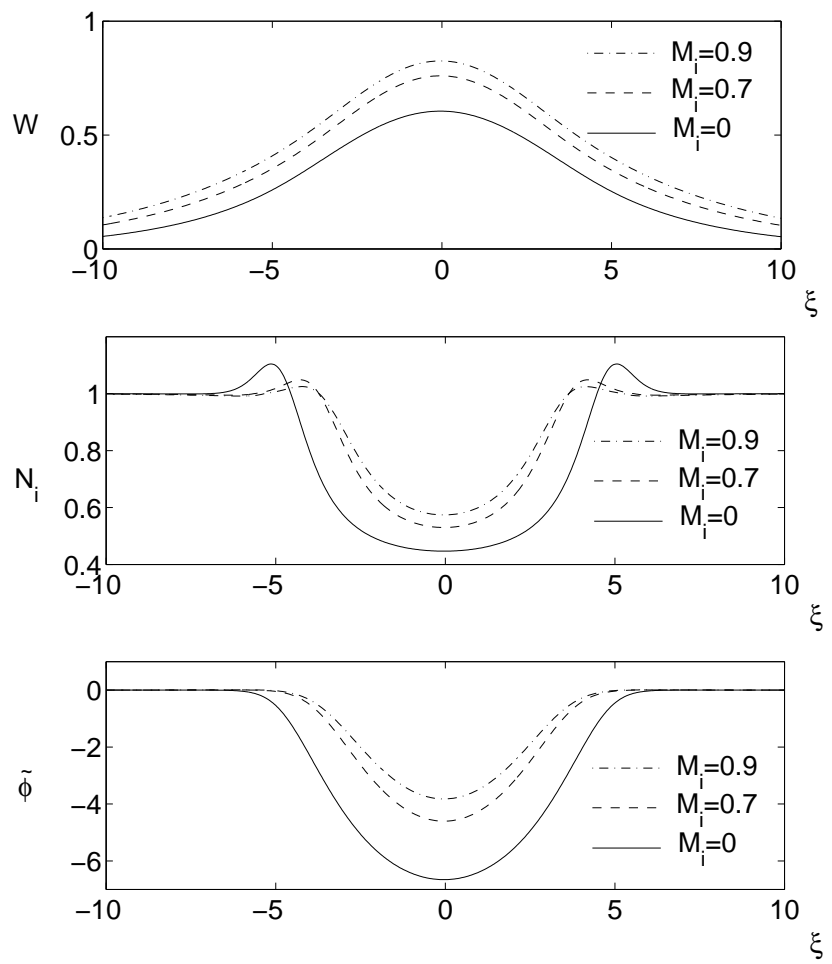

Fig. 8. The electric field (upper panel), the ion density (middle panel) and the potential (lower panel) of ion holes in the presence of large-amplitude Langmuir waves for different Mach numbers $M_{i}$, with $\tau=0.1$ and $\alpha=-1.0$.

This relation overestimates slightly the Langmuir field $W_{0}$ for small $M_{i}$ and underestimates slightly the field for the highest $M_{i}$; see the upper panel in Fig. 8. We assumed a maximum potential of $\tilde{\phi}_{\max }=0.003$. We found that for this set of parameters, the solution had an upper bound $M_{i}=1.25$ for the existence of localized solutions, which is clearly smaller than the existence in the absence of the Langmuir fields. In a more exact mapping of the existence of ion holes, one needs to explore more carefully the relationships between different parameters in Eq. (22), possibly by solving the system of Eqs. (17) and (18) for different cases. Furthermore, the dynamics of the time-dependent system is not explored here,

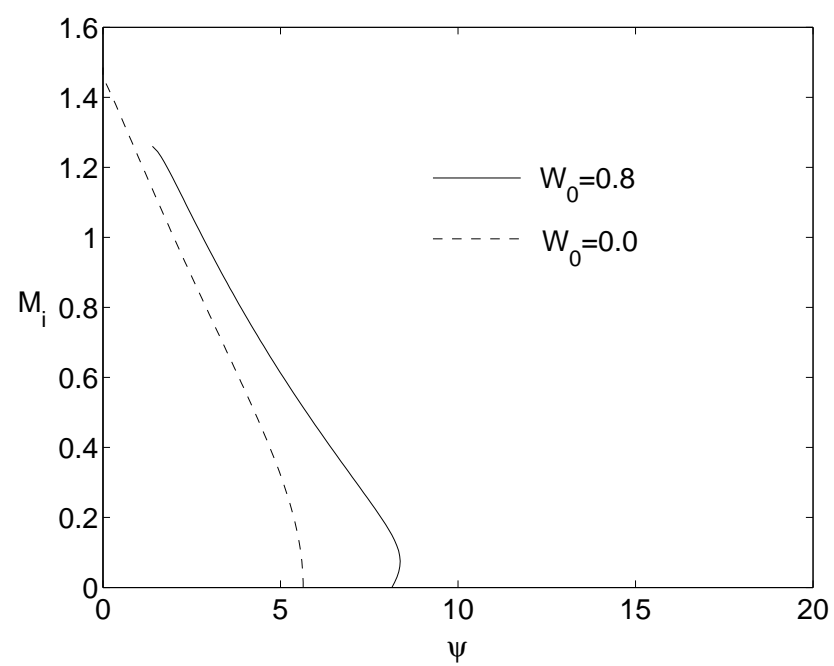

Fig. 9. Numerical solutions of Eq. (22), depicting $\psi\left(=-\tilde{\phi}_{\min }\right)$ versus $M_{i}$ for $W_{0}=0.0$ and $W_{0}=0.8$, with $\tau=0.1$ and $\alpha=$ -1.0. We see that the ion hole loaded with the Langmuir wave electric fields has an upper bound on the Mach number, which is smaller than the one without the Langmuir wave fields.

but is studied by direct simulations of the Vlasov-Poisson system below.

It should be stressed that the properties of the present Langmuir envelope solitons significantly differ from those based on Zakharov's model (1972) which utilizes the fluid ion response for driven (by the Langmuir wave ponderomotive force) ion-acoustic perturbations and yield subsonic density depression accompanied with a positive localized ambipolar potential structure. Furthermore, consideration of a Boltzmann ion density distribution, viz. $N_{i}=\exp (-\tilde{\phi})$, would correspond to the case $M_{i}=0$ and $\alpha=1$ in Eq. (18). Here, as shown in Fig. 10, we have a localized Langmuir wave electric field envelope trapped in a standing ion density cavity. The corresponding slow ambipolar potential is positive and localized.

In the numerical solutions of Eqs. (17) and (18), the second derivatives were approximated by a second-order centered difference scheme (Isaacson and Keller, 1994), and the values of $W$ and $\tilde{\phi}$ were set to zero at the boundaries of the 

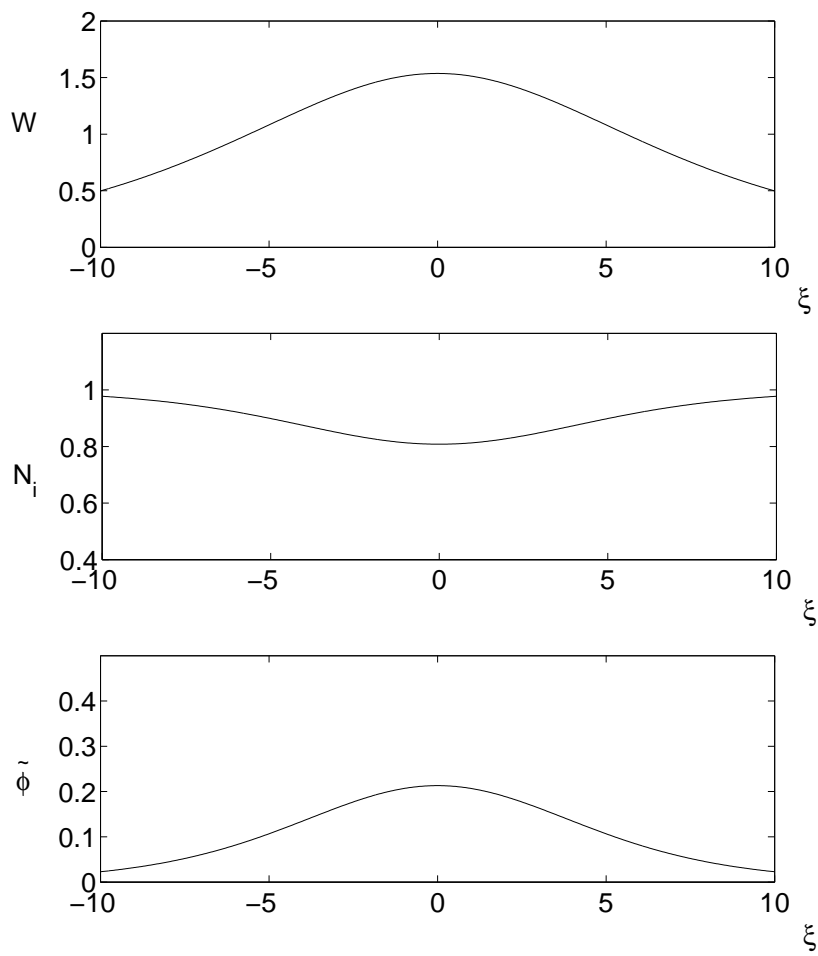

Fig. 10. The Langmuir electric field (upper panel), the ion density (middle panel) and the potential (lower panel) of a Langmuir caviton with a Boltzmann ion distribution for $M_{i}=0, \lambda=0.1, \tau=0.1$ and $\alpha=1.0$.

computational domain at $\xi= \pm 40$. The resulting nonlinear system of equations was solved iteratively. We used 2000 sampling points to resolve the solution.

\subsection{Vlasov simulations of trapped Langmuir waves}

In order to explore the dynamics of trapped Langmuir waves in an ion hole, we have performed a new set of simulations to study the time-dependence of trapped Langmuir wave electric fields. The results are displayed in Fig. 11.

In these simulations, the electron density is initially perturbed so that Langmuir waves are excited around the ion hole centered at $x / r_{D}=0$. We examine the above discussed ion hole with zero-speed (the left panel) and the ion hole having the speed $M_{i}=0.9$ (the right panel). Figure 11 shows that Langmuir waves are trapped in the ion hole, with a maximum wave amplitude at $x / r_{D}=0$. We find that the trapped Langmuir waves are oscillating with a real frequency slightly lower than the electron plasma frequency, $\omega_{r} / \omega_{p e} \approx 0.93$ for the standing ion hole case, and $\omega_{r} / \omega_{p e} \approx 0.98$ for for the moving ion hole case with $M_{i}=0.9$, which is consistent with the linear results listed in Table 1. For the standing hole case, the Langmuir waves are strongly Landau damped, with a damping rate $\gamma \approx 0.03 \omega_{\text {pe }}$, which is an effect not covered by our theoretical model which is based on the electron hydrodynamic model for the Langmuir wave packets. In the moving ion hole case, the damping is significantly weaker
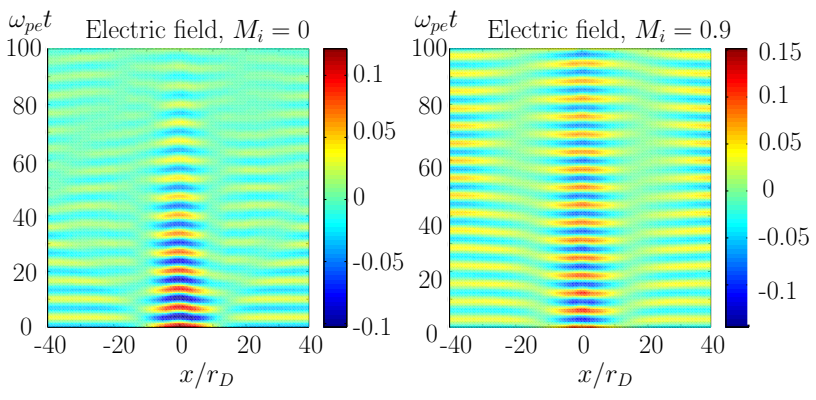

Fig. 11. Normalized Langmuir wave Electric field $E / \sqrt{4 \pi n_{0} T_{i}}$ (the bipolar electric field has been removed from the data) as a function of the normalized space $x / r_{D}$ and time $\omega_{p e} t$. The Langmuir wave is trapped in the ion hole which is initially centered at $x / r_{D}=0$, and moving with the speed $M_{i}=0$ (left panel) and $M_{i}=0.9$ (right panel).

than in the standing hole case. This can be understood in that the frequency shift is smaller for the moving ion hole case, making the scalelength larger for the Langmuir wave envelope, resulting in a smaller Landau damping; some distance away from the ion hole density minimum the potential $\tilde{\phi}$ vanishes, and there the Langmuir wave envelope decreases exponentially with $\xi$ with the scalelength $\sqrt{3 / \lambda}$. A smaller $\lambda$ leads to a larger length-scale, resulting in a weaker Landau damping. In numerical simulations of large-amplitude Langmuir waves, one has to apply an external pump field to sustain the Langmuir field at constant amplitude, since the trapped Langmuir waves are Landau damped. We have observed the following phenomena in Vlasov simulations: High-intensity Langmuir waves are initially trapped inside the ion hole. The ion density well associated with the ion hole deepened, and the ion density surrounding the ion hole is depleted. The Langmuir field trapped in the ion hole accelerated electrons which formed propagating electron BGK waves (electron holes). We could not observe a clear widening of the ion hole predicted by theory. Possible explanations of this discrepancy can be that the electrons are strongly heated in the simulation, which increases the temperature ratio $\tau$, leading to a scenario in which the ion hole tends to become smaller if other parameters are kept constant $(\mathrm{Bu}-$ jarbarua and Schamel, 1981). Further, the theory does not predict how the parameters of an ion hole changes dynamically when the amplitude of the trapped Langmuir envelope changes in time.

\section{The dynamics of nonrelativistic electron holes in plas- mas}

In this section, we study the dynamics of nonrelativistic electron holes in an electron-ion plasma. The shape of the electron hole is not unique but is strongly dependent on the history of its creation. In order to investigate the dynamics of electron holes numerically in a controlled manner, we use Schamel's solution of the stationary Vlasov-Poisson sys- 

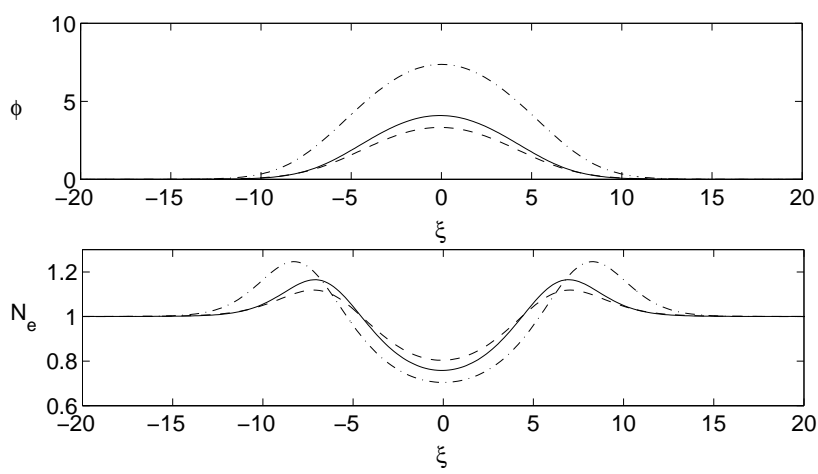

Fig. 12. The potential (upper panel) and the electron density (lower panel), associated with a standing electron hole $\left(M_{e}=0\right)$ with $\beta=-0.7$ (solid lines) and $\beta=-0.5$ (dash-dotted lines), and a moving electron hole with $M_{e}=0.5$ and $\beta=-0.7$ (dashed lines) in plasmas with fixed ion background $\left(N_{i}=1\right)$.

tem to construct initial conditions for our simulations. The Schamel solution is well-behaved in the sense that the solution for the distribution function is continuous and that it goes to a shifted Maxwellian distribution far away from the electron hole where the electric potential vanishes. Following Bujarbarua and Schamel (1981), we prescribe solutions to the electron Vlasov equation in the form of distributions of free and trapped electrons, as given in Eq. (8). We here use $m_{i} / m_{e}=29500$ for oxygen ions. Integrating the untrapped and trapped electron distributions over velocity space, we obtain the electron density

$N_{e}=e^{-M_{e}^{2} / 2}\left[I(\phi)+\kappa\left(\frac{M_{e}^{2}}{2}, \phi\right)+\frac{2 W_{D}\left[(-\beta \phi)^{1 / 2}\right]}{(\pi|\beta|)^{1 / 2}}\right]$,

where $M_{e}=u_{0} / V_{T e}$ is the Mach number, $u_{0}$ is the speed of the electron hole, and $\phi=e \varphi / T_{e}$ is the scaled potential. The special functions $I, \kappa$ and $W_{D}$ are given in Eqs. (14)-(15), respectively. Poisson's equation

$r_{D}^{2} \frac{\partial^{2} \phi}{\partial x^{2}}=N_{e}-N_{i}$

where $N_{e}$ is given by Eq. (26), is solved as a nonlinear boundary value problem where $\phi$ is set to zero far away on each side of the electron hole; a central difference approximation is used for the second derivative in Poisson's equation, leading to a system of nonlinear equations, which is solved iteratively with (a slightly modified) Newton's method.

In Fig. 12, we have plotted the electric potential and electron number density of the electron hole for the case of a fixed ion background, viz. $N_{i}=1$. We note that larger values of $M_{e}$ and $|\beta|$ give smaller maxima $\psi$ of the potential and less deep electron density minima, in agreement with Fig. 3a of Bujarbarua and Schamel (1981). The potentials obtained in Fig. 12 are used to construct the numerical initial conditions for the electron distribution function of electron holes (Bujarbarua and Schamel, 1981), to be used in our Vlasov simulations including the ion dynamics.
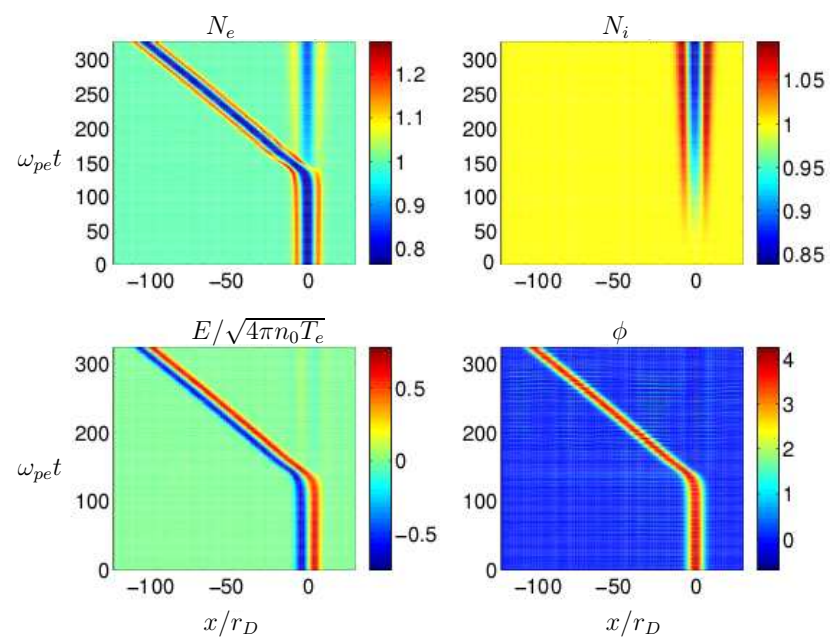

Fig. 13. The electron density (upper left panel), the ion density (upper right panel), the electric field (lower left panel) and the potential (lower right panel) of an initially standing electron hole. The chosen trapping parameter is $\beta=-0.7$.

In Fig. 13 we show the time development of an electron hole initially at rest. As the initial condition for the electron distribution function, we use the parameters $M_{e}=0$ and $\beta=-0.7$ (the solid lines in Fig. 12), with a small local perturbation of the plasma near the electron hole. The perturbation consisted of a Maxwellian population of electrons added to the initial condition for the electron hole, with the same temperature as the background electrons and with the density perturbation of the form $\delta N_{e}=$ $-0.008 \sinh \left(x / 2 r_{D}\right) / \cosh ^{2}\left(x / 2 r_{D}\right)$. The most striking feature, seen at time $t \approx 130 \omega_{\text {pe }}^{-1}$, is that the electron hole "suddenly" starts moving in the negative $x$ direction with a Mach number $M_{e} \approx 0.55$. (The direction of the propagation depends on the perturbation in the initial condition.) The transition seems to happen when the ion density has formed a deep enough cavity. When the electron hole has escaped the ion cavity, the ion density cavity continues to deepen and an electron density cavity is created at the same place, neutralizing the plasma. In Fig. 14, we have repeated the same numerical experiment as in Fig. 13, including the local perturbation of the plasma, but with $\beta=-0.5$, making the electron hole larger. In this case, the electron hole starts moving in the positive $x$ direction at $t \approx 100 \omega_{\text {pe }}^{-1}$, also with a Mach number $M_{e} \approx 0.55$. For both the $\beta=-0.7$ and $\beta=-0.5$ cases, the potential maximum decreases slightly during the transition from standing to moving electron holes, from $\psi=4.5$ to $\psi=3.8$ and from $\psi=7.8$ to $\psi=7.0$, respectively. For $M_{e}=0.55$, the diagram in Fig. 14a of Bujarbarua and Schamel (1981) predicts that the trapping parameter has changed to $\beta \approx-0.67$ and $\beta \approx-0.45$, respectively, for the two cases. We next analyze the distribution of energy in the system. The total energy is conserved exactly in the continuous system and to a high degree in our numerical simulations (Eliasson, 2001), and is distributed between the 

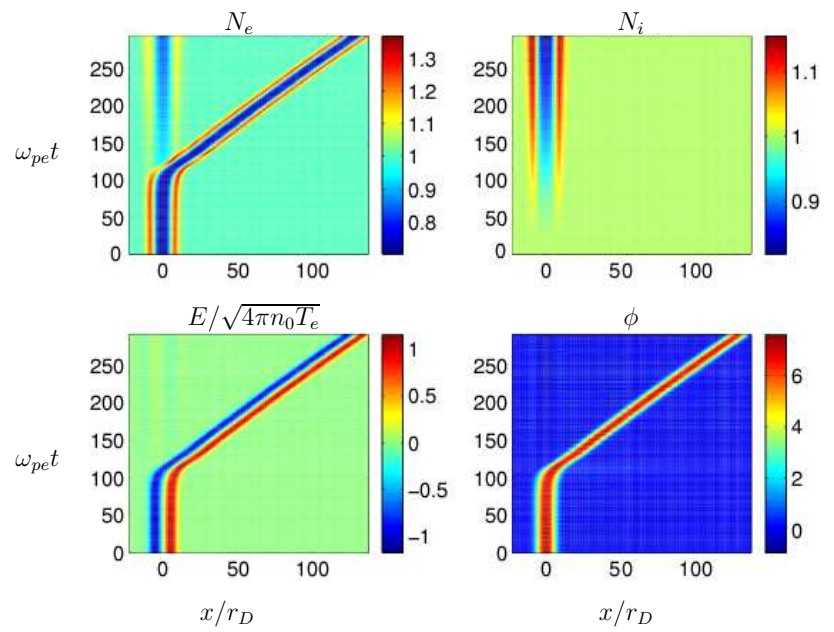

Fig. 14. The electron density (upper left panel), the ion density (upper right panel), the electric field (lower left panel) and the potential (lower right panel) of an initially stationary electron hole. The chosen trapping parameter is $\beta=-0.5$.

kinetic energy of the particles and the potential energy stored in the electric field.

In the upper panel of Fig. 15, we have plotted the potential energy $W_{p o t}=(1 / 2) \int E^{2} d x$ of the system for the two simulation runs, as a function of time. Here, most of the potential energy is stored in the large-amplitude bipolar electric field of the electron hole, while some potential energy is released in high-frequency Langmuir waves. We observe a gradual decrease of the potential energy till the transition time when the electron hole leaves the ion cavity and the potential energy performs large fluctuations $\left(t \approx 130 \omega_{p e}^{-1}\right.$ for the $\beta=-0.7$ case and $t \approx 100 \omega_{p e}^{-1}$ for the $\beta=-0.5$ case), whereafter the potential energy performs high-frequency oscillations attributed to Langmuir waves released in the transition, around a somewhat smaller constant $W_{p o t}$ attributed to the propagating electron hole bipolar electric field. The slow decrease of the potential energy in the initial phase indicates that the positive electrostatic potential of the electron hole accelerates the ions which leave the vicinity of the electron hole. In a homogeneous ion background, the single, steady-state electron hole is associated with a positive potential which traps electrons. Since the positive potential of the electron hole and the negative potential of the ion cavity are competing processes, there could be a problem of the existence of a stationary electron hole if the ion density becomes deep enough. In our case, the electron hole remains stable but escapes the ion cavity. In the lower panel of Fig. 15, we have taken the ion density $N_{i}$ obtained in our Vlasov simulation as an input to Poisson's equation, which we then solve with the electron density given by Eq. (26). The potential of the electron hole is normally positive everywhere when the ions are fixed background. In the presence of a local ion density cavity, the potential may have a slightly negative minimum, and at this point we prescribe a Maxwellian distribution for the untrapped electrons in the same manner as often done by
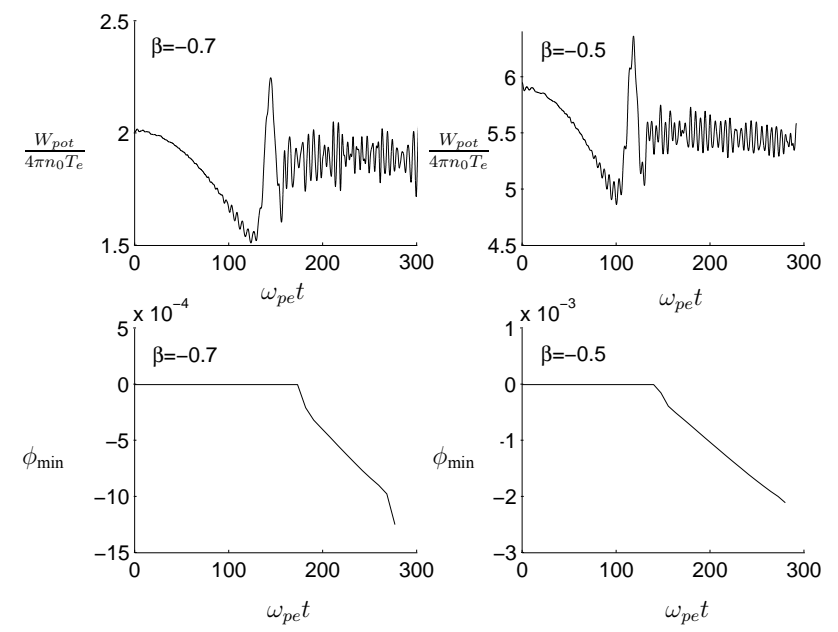

Fig. 15. The total potential energy $W_{\text {pot }}=(1 / 2) \int E^{2} d x$ as a function of time (upper panels) and the minimum of the electrostatic potential (lower panels) for $\beta=-0.7$ (left panels) and $\beta=-0.5$ (right panels), obtained from the model for a stationary electron hole in the presence of a varying ion density, where the ion density is obtained from the Vlasov simulations.

Schamel (1971, 1986). We plotted the potential minimum $\phi_{\min }$ as a function of time, and found that at $t \approx 170 \omega_{p e}^{-1}$ a part of the potential becomes negative. It seems that the acceleration of the electron hole occurs at approximately the same time as a part of the potential for the theoretical model becomes negative. An alternative way of explaining accelerating electron holes is as follows: The positive electron hole potential starts to reflect low energy ions giving rise to a local reduction of $N_{i}$. After a while, at the center of the electron hole, the condition $\phi^{\prime \prime}(x)=N_{e}(x)-N_{i}(x)<0$ for $x$ near zero is no longer met and the standing structure ceases to exist. Then the electron hole is accelerated such that a free ion component exists, giving rise to a free ion density $N_{i f}(x)$ necessary for the maintenance of the inequality near the electron hole center. Figure 13 indicates that indeed $N_{i}(x)=N_{i f}(x) \approx 1$ at the location of the propagating electron hole.

Finally, we have studied interactions between two electron holes with each other and with ions in a longer simulation; see Figs. 16 and 17. The electron holes with $\beta=$ -0.5 and $\beta=-0.7$ were initially placed at $x=-40 r_{D}$ and $x=40 r_{D}$, respectively. A local electron density perturbation was taken to be Maxwellian with the density $\delta N_{e}=-0.08\left\{\sinh \left[\left(x / r_{D}+40\right) / 2\right] / \cosh ^{2}\left[\left(x / r_{D}+40\right) / 2\right]+\right.$ $\left.\sinh \left[\left(x / r_{D}-40\right) / 2\right] / \cosh ^{2}\left[\left(x / r_{D}+-40\right) / 2\right]\right\}$, i.e. the same perturbations as in the single-hole cases, centered at the two electron holes. Here, the electron holes also creates local ion density cavities, and after some time escapes the density cavities at the same times as in the single electron hole cases. This can clearly be seen in Fig. 16, where the two electron holes start moving at $t \approx 100 \omega_{p e}^{-1}$ and $t \approx 130 \omega_{p e}^{-1}$, respectively. At $t \approx 170 \omega_{p e}^{-1}$, the two electron holes collide inelastically and merge into a new electron hole, in accor- 

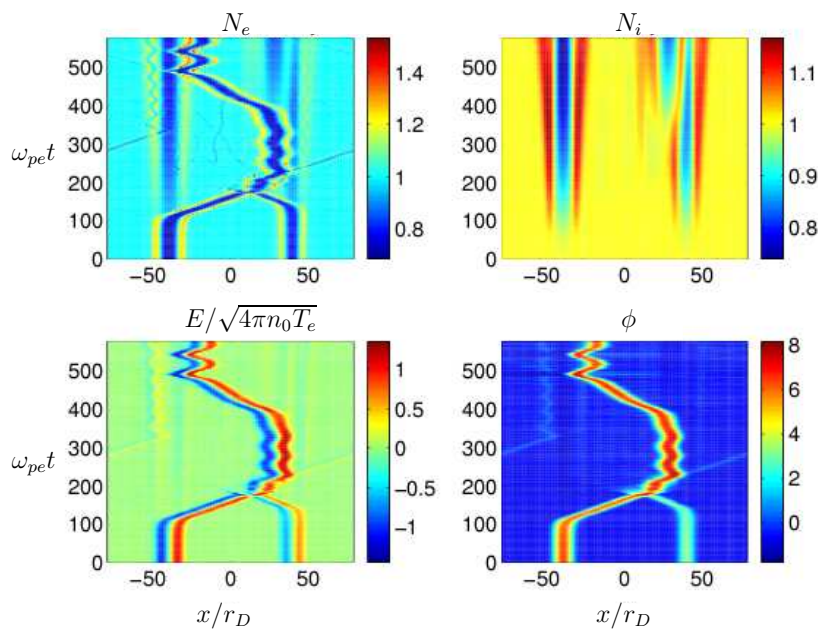

Fig. 16. The electron density (upper left panel), the ion density (upper right panel), the electric field (lower left panel), and the potential (lower right panel) of two electron holes initially placed at $x / r_{D}= \pm 40$. The trapping parameter $\beta=-0.5$ for the left electron hole initially placed at $x / r_{D}=-40$, and $\beta=-0.7$ for the right electron hole placed at $x / r_{D}=40$.

dance with the results of Matsumoto (1994), whereafter the single electron hole propagates slightly in the positive $x$ direction, and becomes trapped at a local ion density maximum at $x \approx 30 r_{D}$; see the upper right panel of Fig. 16 for the ion density and the lower right panel for the electron hole potential. After $t \approx 400 \omega_{p e}^{-1}$, a new ion density cavity is created where the electron hole is centered, and at this time the electron hole is again accelerated in the negative $x$ direction. At $t \approx 480 \omega_{p e}^{-1}$, the moving electron hole again encounters an ion density maximum located at $x \approx-30 r_{D}$, where the electron hole is trapped, performing large oscillations. The electron phase space density is depicted in Fig. 17: The initial condition (upper left panel), the two electron holes having started moving (upper right panel), collisions between the two electron holes (middle left panel), the newly created electron hole trapped at $x=30 r_{D}$ (middle right panel), and the electron hole trapped at $x=-30 r_{D}$ (lower panels). We see that the electron holes remain stable during the acceleration by ion density cavities.

The numerical solutions of the Vlasov-Poisson system were performed with a Fourier method (Eliasson, 2001). We used 500 intervals in $x$ space with the domain $-80 \leq$ $x / r_{D} \leq 80$, and 300 intervals in velocity space. The electron velocity interval was set to $-15.7 \leq v / V_{T e} \leq 15.7$ and the ion velocity interval was set to $-0.118 \leq v / V_{T e} \leq 0.118$. The time-step $\Delta t \approx 0.013 \omega_{p e}^{-1}$ was adapted dynamically to maintain numerical stability.

\section{Relativistic electron holes}

In the present section, we develop a theory for the steady state electron holes in a relativistic plasma. We find that the
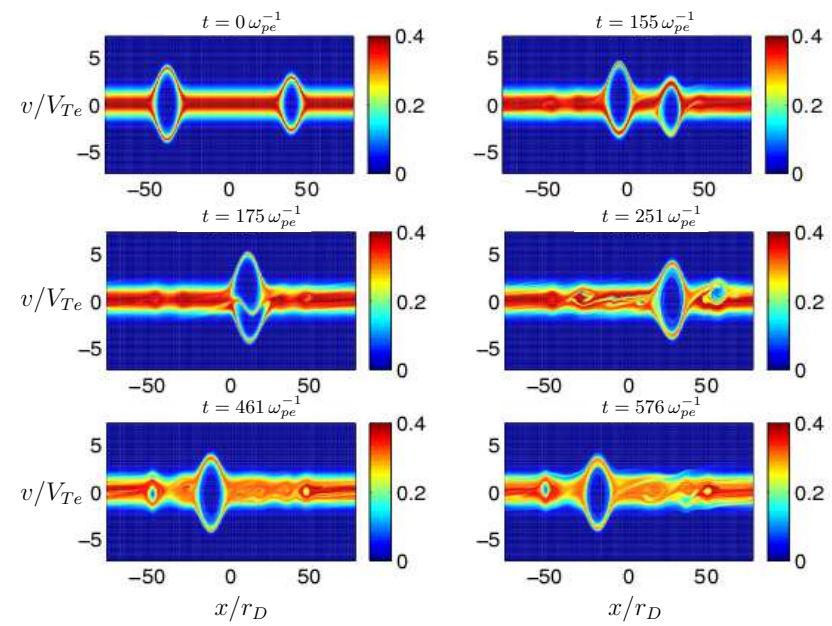

Fig. 17. The electron distribution for two electron holes at $t=$ $0 \omega_{p e}^{-1}$ (upper left panel), $t=155 \omega_{p e}^{-1}$ (upper right panel), $t=$ $175 \omega_{p e}^{-1}$ (middle left panel), $t=251 \omega_{p e}^{-1}$ (middle right panel), $t=$ $461 \omega_{p e}^{-1}$ (lower left panel) and $t=576 \omega_{p e}^{-1}$ (lower right panel). See the associated densities etc. in Fig. 16. Initially, the left electron hole (placed at $x / r_{D}=-40$ ) has a trapping parameter $\beta=-0.5$, while the right electron hole (placed at $x / r_{D}=40$ ) has $\beta=-0.7$.

properties of the electron holes are dramatically changed by the relativistic mass increase of the electrons. Our relativistic simulation studies show that strongly relativistic electron holes are long-lived, and that they survive head-on collisions. We expect that intense electron hole potentials can accelerate electrons to extremely high energies.

The dimensionless relativistic Vlasov-Poisson system, where ions are assumed to form a neutralizing background, is

$\frac{\partial f_{e}}{\partial t}+\frac{p}{\sqrt{1+\mu^{2} p^{2}}} \frac{\partial f_{e}}{\partial x}+\frac{\partial \phi}{\partial x} \frac{\partial f_{e}}{\partial p}=0$,

and

$\frac{\partial^{2} \phi}{\partial x^{2}}=\int_{-\infty}^{\infty} f_{e} d p-1$,

where $\mu=V_{T e} / c$, the distribution function $f_{e}$ has been normalized by $n_{0} / m_{e} V_{T e}, t$ by $\omega_{p e}^{-1}, x$ by $r_{D}$, the momentum $p$ by $m_{e} V_{T e}$, and $\phi$ by $T_{e} / e$. Here, $c$ is the speed of light in vacuum and the nonrelativistic plasma frequency is $\omega_{p e}=\left(4 \pi n_{0} e^{2} / m_{e}\right)^{1 / 2}$. We (the observer) remain in the frame of the bulk plasma while the electron hole is moving with the speed $u_{0}$. Accordingly, we look for solutions of the form $f(p, \xi)$ and $\phi(\xi)$, where $\xi=x-M_{e} t, M_{e}=u_{0} / V_{T e}$ is the Mach number and $u_{0}$ is the constant propagation speed of the electron hole. With this ansatz, the Vlasov-Poisson system (28) and (29) takes the form

$\left(-M_{e}+\frac{p}{\sqrt{1+\mu^{2} p^{2}}}\right) \frac{\partial f_{e}}{\partial \xi}+\frac{d \phi}{d \xi} \frac{\partial f_{e}}{\partial p}=0$, 
and

$\frac{d^{2} \phi}{d \xi^{2}}=\int_{-\infty}^{\infty} f_{e} d p-1$.

Integrating Eq. (30) along its trajectories, one finds the general solution of the form $f_{e}=f_{0}(\mathcal{E})$, where $f_{0}$ is some function of one variable and

$\mathcal{E}=-M_{e} p+\frac{1}{\mu^{2}}\left(\sqrt{1+\mu^{2} p^{2}}-\frac{1}{\gamma_{0}}\right)-\phi$

is a conserved quantity (energy) along the particle trajectories. Here, we have denoted $\gamma_{0}=1 / \sqrt{1-\mu_{0}^{2} M_{e}^{2}}$. At the electron hole, a population of electrons is trapped in a localized positive potential $\phi$, which goes to zero far away from the electron hole. The energy $\mathcal{E}$ has been shifted so that the trapped electrons have a negative energy, while the untrapped (free) electrons have a positive energy.

A condition for the untrapped electrons is that far away from the electron hole, the distribution should connect smoothly to a Jüttner-Synge distribution (de Groot et al., 1980), which restricted to one momentum dimension takes the form

$\tilde{f}_{0}(p)=a_{0} \exp \left[-\frac{1}{\mu^{2}}\left(\sqrt{1+\mu^{2} p^{2}}-1\right)\right]$,

where the normalization constant is

$$
\begin{gathered}
a_{0}=\left\{\int_{-\infty}^{\infty} \exp \left[-\frac{1}{\mu^{2}}\left(\sqrt{1+\mu^{2} p^{2}}-1\right)\right] d p\right\}^{-1} \\
=\frac{\mu \exp \left(-\mu^{-2}\right)}{2 \mathrm{~K}_{1}\left(\mu^{-2}\right)},
\end{gathered}
$$

and $\mathrm{K}_{1}$ is a modified Bessel function of second kind. The weakly relativistic limit corresponds to $\mu \ll 1$ so that $a_{0} \approx$ $\left(1+3 \mu^{2} / 8\right)^{-1} / \sqrt{2 \pi}$ (Gradshteyn and Ryzhik, 1965), and Eq. (33) converges to a non-relativistic Maxwellian distribution when $\mu \rightarrow 0$. In order to impose the above mentioned condition for the free electrons, we use the solution

$f_{e}=f_{0}(\mathcal{E})=\tilde{f}_{0}[\tilde{p}(\mathcal{E})], \quad \mathcal{E}>0$,

where $\tilde{p}(\mathcal{E})$ is a function of the energy such that $\tilde{p}(\mathcal{E}) \rightarrow p$ when $\phi \rightarrow 0$. Such a function can be found with the help of Eq. (32) by setting

$$
-M_{e} \tilde{p}(\mathcal{E})+\frac{1}{\mu^{2}}\left(\sqrt{1+\mu^{2} \widetilde{p}^{2}(\mathcal{E})}-\frac{1}{\gamma_{0}}\right)=\mathcal{E} .
$$

Solving for $\widetilde{p}(\mathcal{E})$, we have

$$
\tilde{p}_{ \pm}(\mathcal{E})=\gamma_{0}^{2} M_{e}\left(\mu^{2} \mathcal{E}+\frac{1}{\gamma_{0}}\right) \pm \frac{\gamma_{0}^{2}}{\mu} \sqrt{\left(\mu^{2} \mathcal{E}+\frac{1}{\gamma_{0}}\right)^{2}-\frac{1}{\gamma_{0}^{2}}}
$$

where $\widetilde{p}_{+}(\mathcal{E})$ and $\widetilde{p}_{-}(\mathcal{E})$ correspond to a modified momentum for free electrons on each side of the trapped electron population in momentum space. Using $\widetilde{p}(\mathcal{E})=\widetilde{p}_{+}(\mathcal{E})$ and $\widetilde{p}(\mathcal{E})=\widetilde{p}_{-}(\mathcal{E})$ in Eq. (34), we obtain the distribution function for the free electrons. In the limit of vanishing energy,
$\mathcal{E}=0^{+}$, we have $\tilde{p}_{ \pm}(0)=\gamma_{0} M_{e}$, and the value of the distribution function is

$\left.f_{e}\right|_{\mathcal{E}=0^{+}}=f_{0}(0)=\tilde{f}_{0}\left(\gamma_{0} M_{e}\right)=a_{0} \exp \left(-\frac{\gamma_{0}-1}{\mu^{2}}\right)$,

which should be matched with the distribution function for the trapped electrons with $\mathcal{E}=0$ in order to obtain a continuous distribution function. For the trapped electrons, we choose a relativistic Maxwell-Boltzmann distribution with a negative "temperature", viz.

$f_{e}=a_{0} \exp \left(-\frac{\gamma_{0}-1}{\mu^{2}}-\beta \mathcal{E}\right), \quad \mathcal{E}<0$,

leading to a vortex distribution for $\beta<0$, a flat top distribution for $\beta=0$ and a shifted Jüttner-Synge distribution for $\beta=1$. Clearly, the separatrix between the free and trapped electron distributions is found where $\mathcal{E}=0$ in Eq. (32). Solving for $p$ in Eq. (32) with $\mathcal{E}=0$, we have

$p_{ \pm}=\gamma_{0}^{2} M_{e}\left(\mu^{2} \phi+\frac{1}{\gamma_{0}}\right) \pm \frac{\gamma_{0}^{2}}{\mu} \sqrt{\left(\mu^{2} \phi+\frac{1}{\gamma_{0}}\right)^{2}-\frac{1}{\gamma_{0}^{2}}}$,

where $p_{-}(\phi)$ and $p_{+}(\phi)$ constitute the limits between the trapped and free electron distributions in momentum space. Using these limits and combining Eqs. (34), (36) and (38), we can now write the full electron distribution function as

$f_{e}= \begin{cases}a_{0} \exp \left[-\frac{1}{\mu^{2}}\left(\sqrt{1+\mu^{2} \widetilde{p}_{+}^{2}(\mathcal{E})}-1\right)\right], & p>p_{+}, \\ a_{0} \exp \left[-\frac{\gamma_{0}-1}{\mu^{2}}-\beta \mathcal{E}\right], & p_{-} \leq p \leq p_{+}, \\ a_{0} \exp \left[-\frac{1}{\mu^{2}}\left(\sqrt{1+\mu^{2} \widetilde{p}_{-}^{2}(\mathcal{E})}-1\right)\right], & p<p_{-},\end{cases}$

where $\mathcal{E}$ is given by Eq. (32). Integrating the distribution function over momentum space we obtain the total electron density as a function of $\phi$, which is calculated selfconsistently by means of Poisson's equation (31).

In order to proceed further and to explore the impact of the relativistic effects on the electron holes for different sets of parameters, we have solved Poisson's equation (31) numerically, where the integration of the trapped and free electron populations has been done with a sum representation of the integrals, and Eq. (31) has been solved as a nonlinear boundary value problem where the potential has been set to zero far away from the electron hole, and the resulting nonlinear system of equations have been solved iteratively with a Newtonlike method. An alternative would be to use the so-called potential method (Schamel, 1971). In Fig. 18 we display the electron hole Mach number $M_{e}$ as a function of the maximum amplitude $\psi$ of the potential for different values of the parameter $\mu$. We see that for larger values of $\mu$, the amplitude of the electron holes drastically increases. Note that the electron hole potential has been normalized by $T_{e} / e$, so the curves in Fig. 18 show pure relativistic effects due to the relativistic mass increase of the electrons. In the non-relativistic limit $(\mu=0)$, we recover the previous results of Bujarbarua and Schamel (1981); see their Fig. 1. The profiles of the 


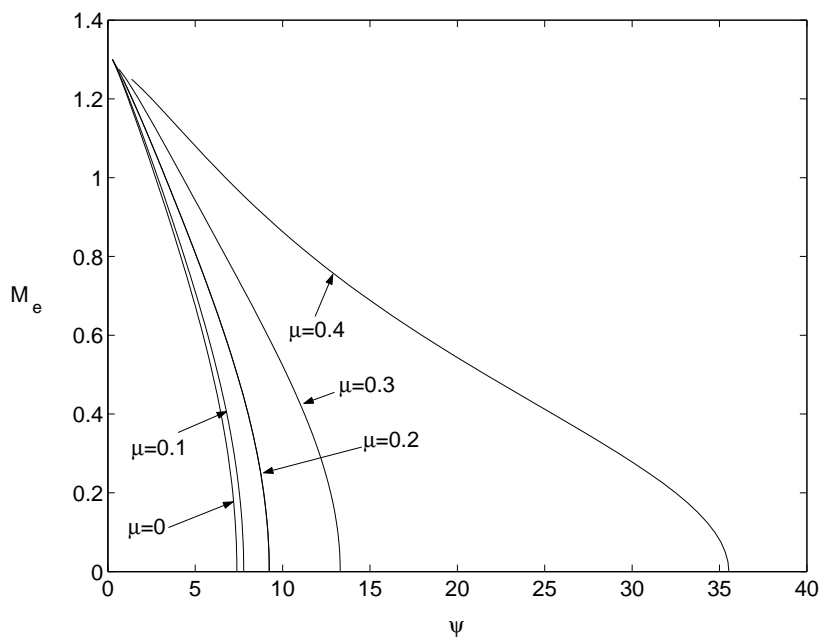

Fig. 18. The Mach number $M_{e}$ of the electron hole as a function of the maximum amplitude $\psi$ of the electron hole potential $\phi$, for a constant trapping parameter $\beta=-0.5$ and for different values of $\mu$.
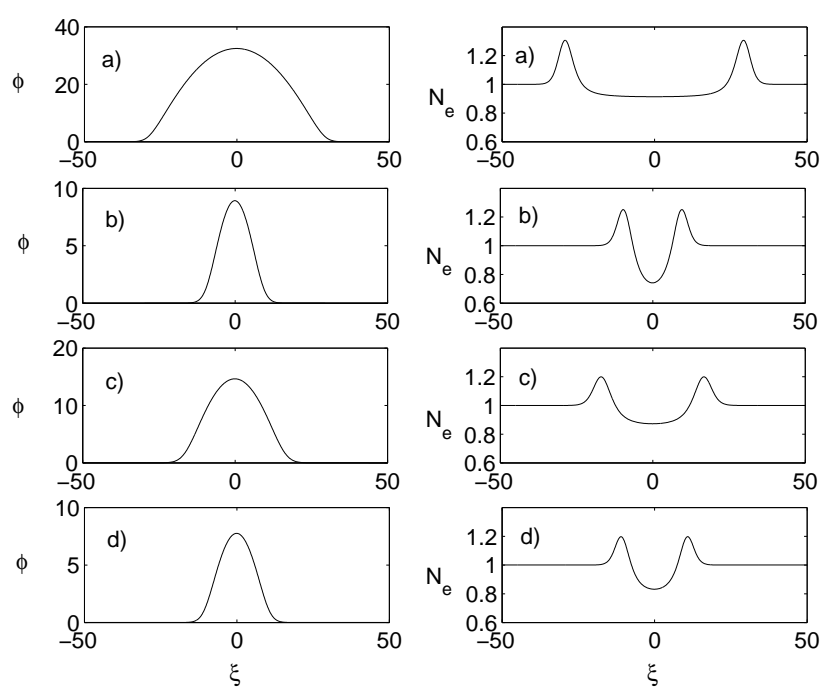

Fig. 19. The potential (left panel) and electron density (right panel) of electron holes with a): $\beta=-0.5, \mu=0.4$ and $M_{e}=0.2, \mathrm{~b}$ ): $\beta=-0.5, \mu=0.2$ and $\left.M_{e}=0.2, \mathrm{c}\right): \beta=-0.5, \mu=0.4$ and $M_{e}=0.7$, and d): $\beta=-0.7, \mu=0.4$ and $M_{e}=0.2$.

electrostatic potential and the electron density are shown in Fig. 19 for a few sets of parameters. To convert the potential to Volts, the values of $\phi$ should be multiplied by the factor $5.1 \times 10^{5} \mu^{2}$; accordingly, the amplitude of the potential for the case a) $(\psi \approx 33, \mu=0.4)$ is $\approx 2.4 \times 10^{6}$ Volts, while for the case b) ( $\psi \approx 9, \mu=0.2)$ it is $\approx 0.18 \times 10^{6}$ Volts. The profiles of the electron holes in phase space is shown in Fig. 20 for the same sets of parameters as in Fig. 19. We have solved the Vlasov-Poisson system (28)-(29) and have investigated interactions between two large-amplitude electron holes. The results are shown in Fig. 21. The left hole has initially the Mach number $M_{e}=0.7$ and the right hole
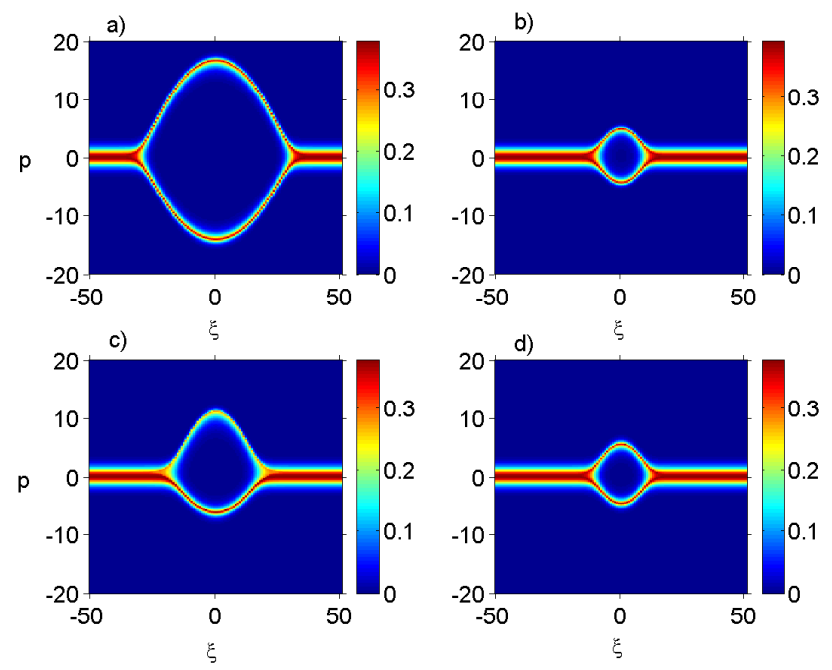

Fig. 20. Phase space plots for electron holes with a): $\beta=-0.5$, $\mu=0.4$ and $\left.M_{e}=0.2, \mathrm{~b}\right): \beta=-0.5, \mu=0.2$ and $\left.M_{e}=0.2, \mathrm{c}\right)$ : $\beta=-0.5, \mu=0.4$ and $M_{e}=0.7$, and d): $\beta=-0.7, \mu=0.4$ and $M_{e}=0.2$, corresponding to the cases a) $-\mathrm{d}$ ) in Fig. 19 .
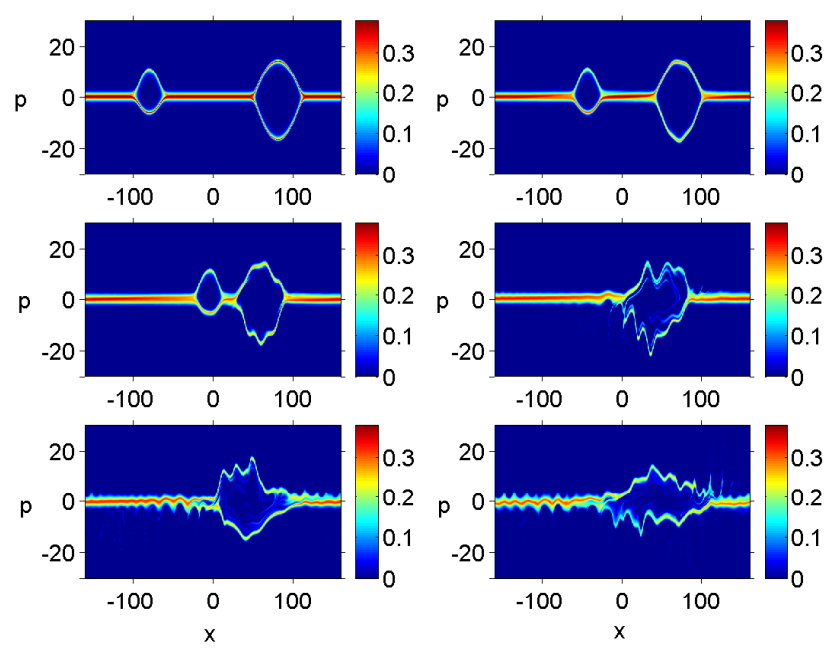

Fig. 21. Interactions between strongly relativistic electron holes. Initially, the parameters are $\mu=0.4$ and $\beta=-0.5$ for both holes, and the left and right holes have the Mach numbers $M_{e}=0.7$ and $M_{e}=-0.2$, respectively, corresponding to the cases a) and c) in Figs. 19 and 20. Time intervals are: $t=0$ (upper left panel), $t=50$ (upper right panel), $t=100$ (middle left panel), $t=150$ (middle right panel), $t=200$ (lower left panel), and $t=250$ (lower right panel).

has a negative Mach number $M_{e}=-0.2$, while both holes have $\beta=-0.5$ and $\mu=0.4$. During head-on collisions, the two holes merge and form a new electron hole, which survives through the rest of the simulation time. As a comparison, shown in Fig. 22, we changed the relativistic factor to $\mu=0.01$, while keeping the same trapping parameter $\beta=-0.5$ and the initial speeds of the holes. In this case, the electron holes are considerably smaller, and do not merge as they did in the strongly relativistic case. The numer- 

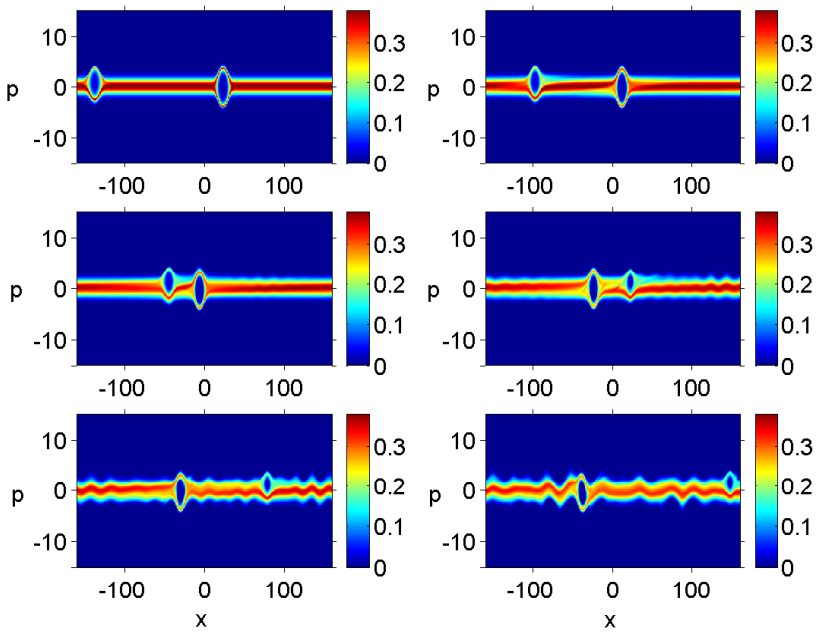

Fig. 22. Interactions between weakly relativistic electron holes. Initially, the parameters are $\mu=0.01$ and $\beta=-0.5$ for both holes, and the left and right holes have the Mach number $M_{e}=0.7$ and $M_{e}=-0.2$, respectively. Times: $t=0$ (upper left panel), $t=50$ (upper right panel), $t=100$ (middle left panel), $t=150$ (middle right panel), $t=200$ (lower left panel) and $t=250$ (lower right panel).

ical method used in our simulations was a pseudo-spectral method to approximate the $x$-derivatives, a fourth-order compact Padé scheme to approximate the $p$ derivative, and the fourth-order Runge-Kutta method for the time stepping. The integral over $p$ space in Eq. (29) was done with a simple sum representation of the integral.

5.1 Trapping of electromagnetic waves in relativistic electron holes

We here present fully relativistic nonlinear theory and computer simulations for intense electromagnetic envelope solitons in a relativistically hot electron plasma, by adopting the Maxwell-Poisson-relativistic Vlasov system which accounts for relativistic electron mass increase in the electromagnetic fields and the relativistic radiation ponderomotive force, in addition to trapped electrons which support the driven relativistic electron holes. The importance of trapped electrons has also been recognized (Montgomery, 2001) in the context of stimulated Compton scattering of laser light off electron quasi-modes in a non-relativistic situation. However, our objective here is to consider nonlinear interactions between intense electromagnetic waves and relativistic electron holes. For this purpose, we derive the electromagnetic wave equation by including the nonlinear current density arising from the coupling of the relativistic electron quiver velocity and the density variation of the relativistic electron holes. The latter are modified by the relativistic ponderomotive force. We find that such nonlinear interactions between electromagnetic waves and relativistic electron holes produce intense electromagnetic envelope solitons composed of localized light wave envelope and large amplitude localized posi- tive electric potential distributions. The latter may contribute to accelerating electrons to extremely high energies. We also present numerical studies of the dynamics of interacting electromagnetic envelope solitons by means of a fully relativistic Vlasov simulations. Interesting nonlinear features of laser light intensification and corresponding density cavitation have been observed.

We present the relevant equations describing the action of intense laser light on the electrons in a relativistically hot collisionless plasma, as well as the electromagnetic wave equation accounting for the relativistic mass increase of the electrons and the electron density modification due to the radiation relativistic ponderomotive force (Shukla, 1986)

$F=-m_{e} c^{2} \frac{\partial \gamma}{\partial z}$

where

$\gamma=\left(1+\frac{p^{2}}{m_{e}^{2} c^{2}}+\frac{e^{2}|\mathbf{A}|^{2}}{m_{e}^{2} c^{4}}\right)^{1 / 2}$

is the relativistic gamma factor. Here, $p$ is the $x$ component of the electron momentum, and we have used that the perpendicular (to the $x$ direction) momentum $p_{\perp}=e \mathbf{A} / c$, where $\mathbf{A}$ is the vector potential of the circularly polarized electromagnetic waves. The dynamics of the coupled electromagnetic waves and relativistic electron holes with immobile ions is governed by

$$
\begin{aligned}
& \frac{\partial^{2} \mathbf{A}}{\partial t^{2}}-\frac{1}{\mu^{2}} \frac{\partial^{2} \mathbf{A}}{\partial x^{2}}+\int_{-\infty}^{\infty} \frac{f_{e}}{\gamma} d p \mathbf{A}=0, \\
& \frac{\partial f_{e}}{\partial t}+\frac{p}{\gamma} \frac{\partial f_{e}}{\partial x}+\frac{\partial\left(\phi-\gamma / \mu^{2}\right)}{\partial x} \frac{\partial f_{e}}{\partial p}=0,
\end{aligned}
$$

and

$\frac{\partial^{2} \phi}{\partial x^{2}}=\int_{-\infty}^{\infty} f_{e} d p-1$,

where $\mathbf{A}$ is normalized by $m_{e} c / e, \phi$ by $T_{e} / e, p$ by $m_{e} V_{T e}$ and $x$ by $r_{D}$. We have denoted $\gamma=\left(1+\mu^{2} p^{2}+|\mathbf{A}|^{2}\right)$.

Far away from the relativistic electron hole, where $\phi=$ $|\mathbf{A}|=0$, the electrons are assumed to obey a Jüttner-Synge distribution function (de Groot et al., 1980)

$\tilde{f}_{0}(p)=a_{0} \exp \left[-\frac{1}{\mu^{2}}\left(\sqrt{1+\mu^{2} p^{2}}-1\right)\right]$,

where the normalization constant is

$$
\begin{aligned}
a_{0}=\left\{\int_{-\infty}^{\infty} \exp \right. & {\left.\left[-\frac{1}{\mu^{2}}\left(\sqrt{1+\mu^{2} p^{2}}-1\right)\right]\right\}^{-1} } \\
= & \frac{\mu \exp \left(-\mu^{-2}\right)}{2 K_{1}\left(\mu^{-2}\right)} .
\end{aligned}
$$

For the Jüttner-Synge distribution function, the last term in the left-hand side of Eq. (43) takes the value

$$
\int_{-\infty}^{\infty} \frac{\widetilde{f}_{0}(p)}{\sqrt{1+\mu^{2} p^{2}}}=\frac{K_{0}\left(\mu^{-2}\right)}{K_{1}\left(\mu^{-2}\right)} \equiv \Omega_{p}^{2},
$$


where $K_{0}$ and $K_{1}$ are the modified Bessel functions of second kind. The frequency $\Omega_{p}$ represents the normalized (by $\omega_{p e}$ ) relativistic plasma frequency at equilibrium.

We now investigate the properties of driven relativistic electron holes which move with a constant speed. Accordingly, we use the ansatz $f(p, \xi)$ for the relativistic electron distribution function, and assume that $\phi$ and $|A|^{2}$ depend on $\xi$ only, where $\xi=x-M_{e} t$. Then, Eqs. (44) and (45) take the form

$$
\left(-M_{e}+\frac{p}{\gamma}\right) \frac{\partial f_{e}}{\partial \xi}+\frac{\partial\left(\phi-\gamma / \mu^{2}\right)}{\partial \xi} \frac{\partial f_{e}}{\partial p}=0,
$$

and

$\frac{d^{2} \phi}{d \xi^{2}}=\int_{-\infty}^{\infty} f_{e} d p-1$

respectively. The general solution of Eq. (48) is $f_{e}=f_{0}(\mathcal{E})$, where $f_{0}$ is some function of one variable and

$\mathcal{E}=-M_{e} p+\frac{1}{\mu^{2}}\left(\gamma-\frac{1}{\gamma_{0}}\right)-\phi$

is the energy integral. Here, we have denoted $\gamma_{0}=$ $1 / \sqrt{1-\mu^{2} M_{e}^{2}}$. We note that trapped electrons have negative energy while untrapped (free) electrons have positive energy.

In in the slowly varying envelope approximation, viz. $\mathbf{A}=$ $(1 / 2) A(x, t)(\widehat{\mathbf{y}}+i \widehat{\mathbf{z}}) \exp \left(-i \omega_{0} t+i k_{0} x\right)+$ complex conjugate, Eq. (43) can be written as

$2 i \omega_{0}\left(\frac{\partial A}{\partial t}+v_{g} \frac{\partial A}{\partial x}\right)+\frac{1}{\mu^{2}} \frac{\partial^{2} A}{\partial x^{2}}$

$-\left(\int_{-\infty}^{\infty} \frac{f_{e}}{\gamma} d p-\Omega_{p}^{2}\right) A=0$,

where $\omega_{0}=\left(\Omega_{p}^{2}+k_{0}^{2} / \mu^{2}\right)^{1 / 2}$ is the electromagnetic wave frequency, $v_{g}=k_{0} / \mu^{2} \omega_{0}$ is the group velocity, and $\gamma=$ $\sqrt{1+\mu^{2} p^{2}+|A|^{2}}$. Introducing $A=W(\xi) \exp (-i \Theta t+$ $i K x$ ) into Eq. (51), where $W$ is a real-valued function, we obtain $K=\mu^{2} \omega_{0}\left(M_{e}-v_{g}\right)$ and

$\frac{d^{2} W}{d \xi^{2}}-\lambda W-\mu^{2}\left(\Omega^{2}-\Omega_{p}^{2}\right) W=0$,

where $\lambda=-2 \omega_{0} \mu^{2} \Theta+\mu^{4} \omega_{0}^{2}\left(M_{e}^{2}-v_{g}^{2}\right)$ represents a nonlinear frequency shift, and the gamma factor becomes $\gamma=$ $\sqrt{1+\mu^{2} p^{2}+W^{2}}$. Here, $\Omega^{2}=\int_{-\infty}^{\infty}\left(f_{e} / \gamma\right) d p$ represents the square of the local electron plasma frequency that accounts for the relativistic electron mass increase in the electron hole potential and the electromagnetic wave fields.

A condition for the untrapped electron is that far away from the relativistic electron hole, the electron distribution function should smoothly connect to the Jüttner-Synge distribution function. In order to impose this condition for the free electrons, we use the solution

$f_{e}=f_{0}(\mathcal{E})=\tilde{f_{0}}[\tilde{p}(\mathcal{E})], \quad \mathcal{E}>0$, where $\tilde{p}(\mathcal{E})$ is a function of the energy such that $\widetilde{p}(\mathcal{E}) \rightarrow p$ when $W \rightarrow 0$ and $\phi \rightarrow 0$. Such a function can be found with the help of the energy integral by setting

$$
-M_{e} \tilde{p}(\mathcal{E})+\frac{1}{\mu^{2}}\left(\sqrt{1+\mu^{2} \widetilde{p}^{2}(\mathcal{E})}-\frac{1}{\gamma_{0}}\right)=\mathcal{E} .
$$

Solving for $\widetilde{p}(\mathcal{E})$, we have

$\tilde{p}_{ \pm}(\mathcal{E})=\gamma_{0}^{2} M_{e}\left(\mu^{2} \mathcal{E}+\frac{1}{\gamma_{0}}\right) \pm \frac{\gamma_{0}^{2}}{\mu} \sqrt{\left(\mu^{2} \mathcal{E}+\frac{1}{\gamma_{0}}\right)^{2}-\frac{1}{\gamma_{0}^{2}}}$,

where $\widetilde{p}_{+}(\mathcal{E})$ and $\widetilde{p}_{-}(\mathcal{E})$ correspond to a modified momentum for free electrons on each side of the trapped electron population in momentum space. Using $\widetilde{p}(\mathcal{E})=\widetilde{p}_{+}(\mathcal{E})$ and $\widetilde{p}(\mathcal{E})=\widetilde{p}_{-}(\mathcal{E})$ in Eq. $(53)$, we obtain the distribution function for free electrons. In the limit of vanishing energy, $\mathcal{E}=0^{+}$, we have $\widetilde{p}_{ \pm}(0)=\gamma_{0} M_{e}$, and the value of the distribution function is

$$
\left.f_{e}\right|_{\mathcal{E}=0^{+}}=f_{0}(0)=\tilde{f}_{0}\left(\gamma_{0} M_{e}\right)=a_{0} \exp \left[-\left(\gamma_{0}-1\right) / \mu^{2}\right] \text {, }
$$

which should be matched with the distribution function for the trapped electrons with $\mathcal{E}=0$ in order to obtain a continuous distribution function. For the trapped electrons, we choose a relativistic Maxwell-Boltzmann distribution with a negative "temperature," viz.

$f_{e}=a_{0} \exp \left(-\frac{\gamma_{0}-1}{\mu^{2}}-\beta \mathcal{E}\right), \quad \mathcal{E}<0$.

The separatrix between the free and trapped electron distributions is found where $\mathcal{E}=0$ in the energy integral. Solving for $p$ in the energy integral with $\mathcal{E}=0$, we have

$p_{ \pm}=\gamma_{0}^{2} M_{e}\left(\mu^{2} \phi+\frac{1}{\gamma_{0}}\right) \pm \frac{\gamma_{0}^{2}}{\mu} \sqrt{\left(\mu^{2} \phi+\frac{1}{\gamma_{0}}\right)^{2}-\frac{1+W^{2}}{\gamma_{0}^{2}}}$,

where $p_{-}$and $p_{+}$constitute the limits between the trapped and free electron distributions in momentum space. Using these limits and combining Eqs. (53), (55) and (57), we can now write

$f_{e}= \begin{cases}a_{0} \exp \left[-\frac{1}{\mu^{2}}\left(\sqrt{+\mu^{2} \widetilde{p}_{+}^{2}(\mathcal{E})}-1\right)\right], & p>p_{+}, \\ a_{0} \exp \left[-\frac{\gamma_{0}-1}{\mu^{2}}-\beta \mathcal{E}\right], & p_{-} \leq p \leq p_{+}, \\ a_{0} \exp \left[-\frac{1}{\mu^{2}}\left(\sqrt{1+\mu^{2} \widetilde{p}_{-}^{2}(\mathcal{E})}-1\right)\right], & p<p_{-} .\end{cases}$

Integrating the distribution function (59) over the momentum space, we obtain the total electron number density as a function of $\phi$ and $W$, which are calculated self-consistently by means of the Poisson and Schrödinger equations, respectively.

Figure 23 exhibits the influence of intense electromagnetic waves on relativistic electron holes, described by the coupled system of Eqs. (49) and (52). In the Schrödinger equation (52), $\lambda$ represents the eigenvalue, and the square of the local electron plasma frequency, $\Omega^{2}$, enters as a "potential." We 


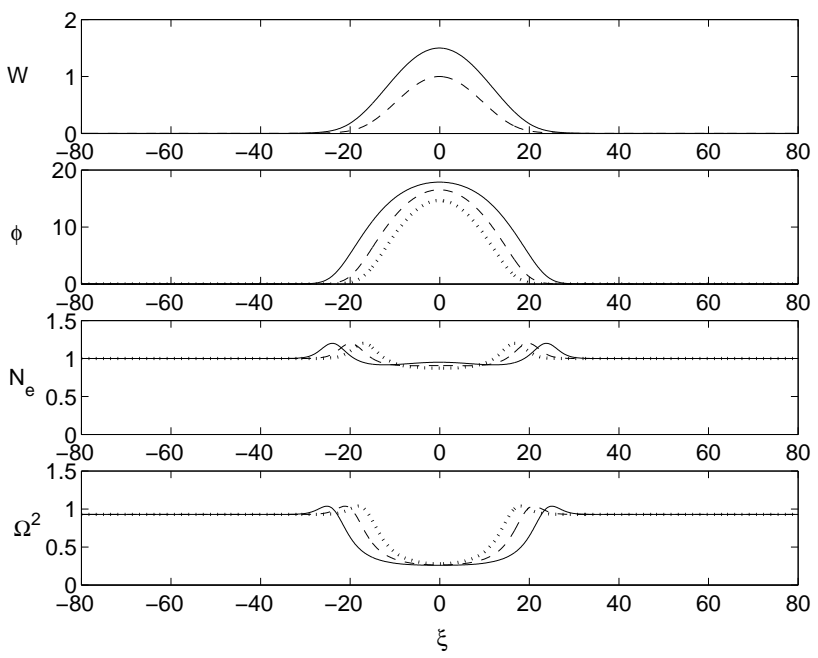

Fig. 23. Large-amplitude trapped electromagnetic wave envelope (upper panel), the potential (second panel), the electron number density (third panel), and the square of the local electron plasma frequency (lower panel) for large amplitude electromagnetic waves with a maximum amplitude of $W_{\max }=1.5$ (solid lines) and $W_{\max }=1.0$ (dashed lines), and as a comparison a relativistic electron hole with small-amplitude electromagnetic waves which have $W_{\max } \ll 1$ (dotted lines). The nonlinear frequency shift for the $W_{\max }=1.5$ case is $\lambda=0.099$, and for the $W_{\max }=1.0$ case it is $\lambda=0.095$, to be compared with the small-amplitude case which has $\lambda=0.088$. Parameters are: $M_{e}=0.7, \mu=0.4$, and $\beta=-0.5$.

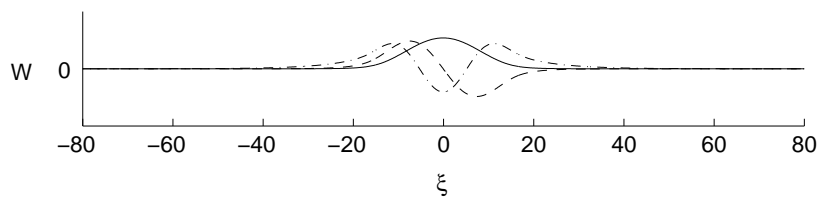

Fig. 24. Small-amplitude trapped electromagnetic waves in a relativistic electron hole. Upper panel: Three eigenstates of trapped electromagnetic waves, corresponding to the eigenvalues $\lambda_{1}=$ 0.088 (solid line), $\lambda_{2}=0.053$ (dashed line) and $\lambda_{3}=0.013$ (dashdotted line). The parameters used are the same as for the dotted lines in Fig. 23.

see that for larger electromagnetic fields $W$, the relativistic electron hole potential $\phi$ becomes larger and the relativistic electron hole wider, admitting larger eigenvalues $\lambda$. This can be explained in that the relativistic ponderomotive force of localized electromagnetic waves pushes the electrons away from the center of the relativistic electron hole, leading to an increase of the electrostatic potential distribution and a widening of the relativistic electron hole. We see that the depletion of the electron density in the relativistic electron hole is only minimal, while the local electron plasma frequency $\Omega$ is strongly reduced owing to the increased mass of the electrons that are accelerated by the relativistic electron hole potential. The linear trapping of electromagnetic waves in rela-
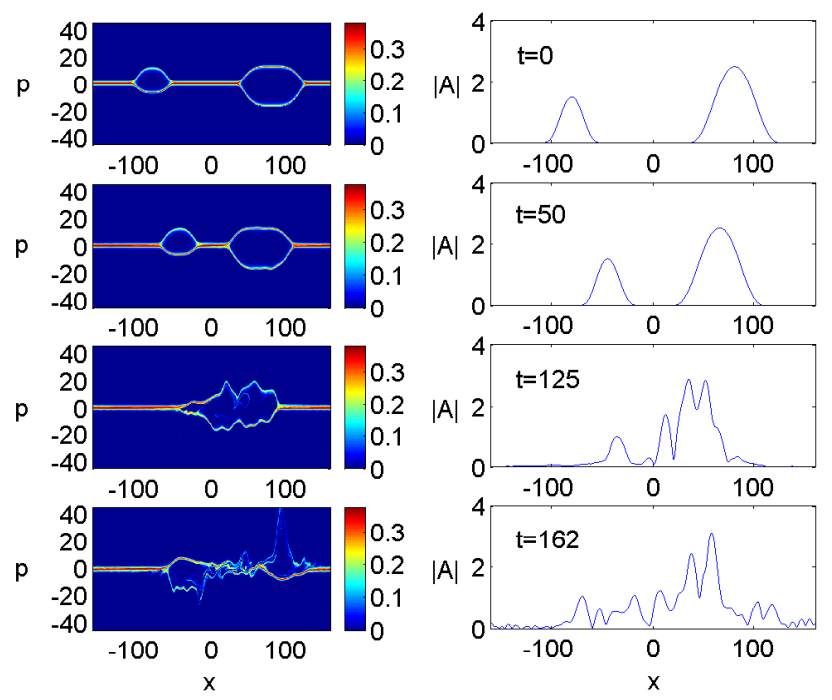

Fig. 25. Phase space plots of the electron distribution function $f_{e}$ (left panels) and the amplitude of the vector potential A (right panels) for $t=0, t=50, t=125$ and $t=162$.
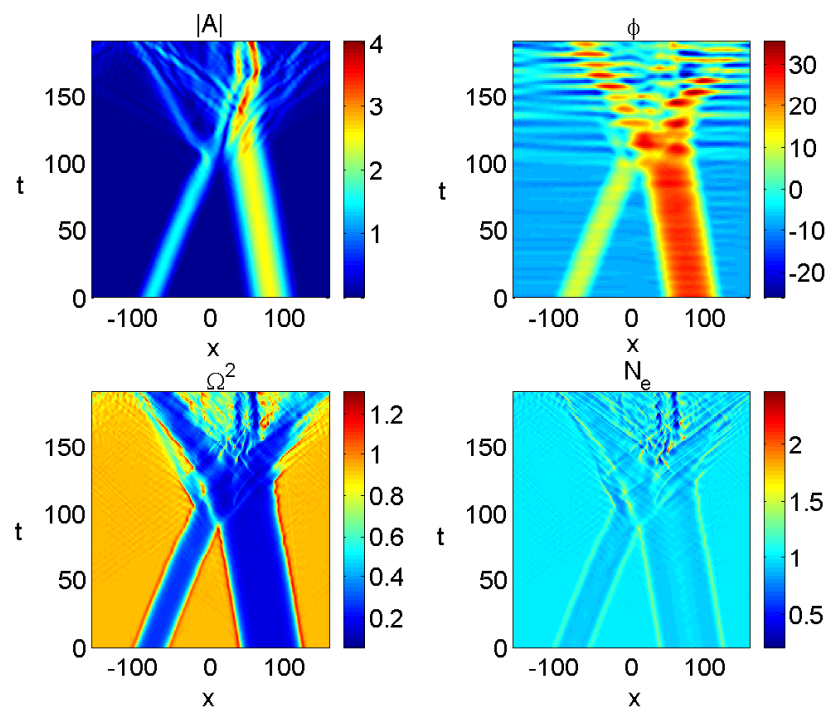

Fig. 26. The amplitude of the vector potential (upper left panel), potential (upper right panel), squared local plasma frequency (lower left panel) and electron density (lower right panel) for two colliding relativistic electron holes.

tivistic electron holes is displayed in Fig. 24, where we have assumed a zero electromagnetic field $(W=0)$ in the expression for $\gamma$ used in Eq. (52) and in the energy integral. The eigenvalue problem admits a discrete set of localized eigenfunctions with positive eigenvalues, and in this case we found two even and one odd eigenfunction corresponding to three different eigenvalues. Equation (52) has been solved as a linear eigenvalue problem for $W$, where the amplitude $W_{\max }$ of the electromagnetic waves was kept fixed, to obtain new val- 
ues on $W$ and $\lambda$. Then, the procedure of solving for $\phi$ and $W$ was repeated until convergence. The second derivatives were approximated with a second-order centered scheme with the function values set to zero at the boundaries.

In order to study the dynamics of interacting electromagnetic envelope solitons composed of localized electromagnetic waves and relativistic electron holes, we have solved the time-dependent, relativistic Vlasov equation (44) together with the Schrödinger equation (51) numerically. The results are displayed in Figs. 25 and 26. As an initial condition to the simulation, we used solutions to the quasi-stationary equations described above, where the left electron hole initially has the speed $M_{e}=0.7$ and is loaded with electromagnetic waves with $W_{\max }=1.5$, while the right electron hole has the speed $M_{e}=-0.3$, and is loaded with electromagnetic waves with $W_{\max }=2.5$. Further, we used $k_{0}=v_{g}=0$ in the initial condition for $A$ and in the solution of Eq. (51). In Fig. 25 we show the phase space distribution of the electrons and the electromagnetic field amplitude at different times. We see that the relativistic electron holes loaded with trapped electromagnetic waves collide, merge and then split into two electron holes, while there are a few strongly peaked electromagnetic wave envelopes at $x \approx 70$ remaining after the splitting of the relativistic electron hole, and where a population of electrons has been accelerated to large energies. The time development of the electromagnetic wave amplitudes, relativistic electron hole potential, the squared local plasma frequency and the electron number density is shown in Fig. 26. We observe collision and splitting of the relativistic electron holes and the creation of localized electromagnetic solitary waves at $z \approx 70$; clearly visible in the left two panels at $t>150$. The electromagnetic solitary waves are created by the combined action of the relativistic electron mass increase and relativistic ponderomotive force of localized electromagnetic waves, which have been further intensified due to nonlinear interactions where the collapsing relativistic electron hole has deposited its energy into the plasma.

\section{Conclusions}

In this paper, we have presented a review of recent findings related to the dynamics of electron and ion holes in a collisionless plasma. We find that interacting (colliding) ion holes may create non-Maxwellian electron distributions due to the acceleration of electrons in the negative ion hole potential. This can create streams of accelerated electrons before and during the ion hole collisions, and a flat-topped electron distribution between the ion holes, after collisions. The accelerated streams of electrons can excite high-frequency Langmuir waves due to a streaming instability, and Langmuir waves can be trapped in the density well of the ion hole, as described by a nonlinear Schrödinger equation for the Langmuir field, coupled nonlinearly with the Poisson equation for the slow timescale ion hole potential response. Electron holes in a plasma with mobile ions show an interesting dynamics when the speed of the electron hole is small enough. A standing electron hole accelerates locally the ions due to the positive electron hole potential. The self-created ion density cavity, on the other hand, accelerates the electron hole, which leaves the ion cavity and propagates with a constant speed. The propagating electron hole can be trapped at ion density maxima, where it again accelerates the ions locally and a new ion density cavity is formed, etc. Thus, both ion and electron holes show an interesting and complex dynamics during their interaction with the background plasma, with a variety of couplings between fast and slow timescales. Knowledge of dynamically evolving electron and ion holes and their interaction with the background plasma is required for understanding the properties of localized electric pulses in the Earth's auroral zone and in the magnetosphere.

We have also presented a theory for relativistic electron holes and their interactions with high-amplitude electromagnetic fields, taking into account the relativistic electron mass increase in a hot plasma. Due to the relativistic effect, the size of the electron hole as well as the amplitude of the electron hole potential increase dramatically with increasing relativistic electron temperature. Large-amplitude electromagnetic fields also modify the electron hole owing to the relativistic ponderomotive force and the electron mass increase due to the relativistic quivering velocity in the highfrequency electromagnetic fields. Simulation studies of interactions between relativistic electron holes show that they interact in a complex fashion, and may merge when they collide, resulting in accelerated particles and a release of the electromagnetic radiation. We stress that the present results should help to understand the salient features of localized intense electromagnetic and electrostatic pulses in relativistically hot plasmas, such as those in inertial confinement fusion as well as in compact astrophysical objects in which gamma-ray bursts are produced by acceleration of electrons to extremely high energies.

Acknowledgements. This work was partially supported by the Deutsche Forschungsgemeinschaft (Bonn) through the Sonderforschungsbereich 591 "Universelles Verhalten Gleichgewichtsferner Plasmen, International Space Science Institute at Bern, as well as by the European Commission (Brussels) through Contract No. HPRN-CT-2001-00314 for carrying out the task of the research training network entitled "Turbulent Boundary Layers in Geospace Plasmas". The Swedish National Supercomputer Centre (NSC) provided the computer resources to carry out this work.

Edited by: J. F. McKenzie

Reviewed by: two referees

\section{References}

Abramowitz, M. and Stegun, I. A.: Handbook of Mathematical Functions, Dover, New York, 1972.

Abbasi, H., Tsintsadze, N. L., and Tskhakaya, D. D.: Influence of particle trapping on the propagation of ion cyclotron waves, Phys. Plasmas, 6, 2373-2379, 1999.

Bale, S. D., Kellogg, P. J., Larson, D. E., Lin, R. P., Goetz, K., and Lepping, R. P.: Bipolar electrostatic structures in the shock tran- 
sition region: Evidence of electron phase space holes, Geophys. Res. Lett., 25, 2929-2932, 1998.

Bale, S. D., Hull, A., Larson, D. E., Lin, R. P., Muschietti, L., Kel$\operatorname{logg}$, P. J., Goetz, K., and Monson, S. J.: Electrostatic turbulence and Debye-scale structures associated with electron thermalization at collisionless shocks, Astrophys. J., 575, L25-L28, 2002.

Bingham, R.: Accelerator physics: In the wake of success, Nature (London), 424, 258-259, 2003.

Bingham, R., Mendonça, J. T., and Shukla, P. K.: Plasma based charged-particle accelerators, Plasma Phys. Control. Fusion, 46, R1-R23, 2004.

Borghesi, M., Bulanov, S., Campbell, D. H., Clarke, R. J., Esirkepov, T. Zh., Galimberti, M., Gizzi, L. A., MacKinnon, A. J., Naumova, N. M., Pegoraro, F., Ruhl, H., Schiavi, A., and Willi, O.: Macroscopic Evidence of Soliton Formation in Multiterawatt Laser-Plasma Interaction, Phys. Rev. Lett., 88, 135002/14, 2002.

Boström, R., Gustafsson, G., Holback, B., Holmgren, G., Koskinen, H., and Kintner, P.: Characteristics of solitary waves and weak double layers in the magnetospheric plasma, Phys. Rev. Lett., 61, 82-85, 1988.

Bujarbarua, S. and Schamel, H.: Theory of electron and ion holes, J. Plasma Phys., 25, 515-529, 1981.

Bulanov, S. V., Esirkepov, T. Zh., Naumova, N. M., Pegoraro, F., and Vshivkov, V. A.: Solitonlike Electromagnetic Waves behind a Superintense Laser Pulse in a Plasma, Phys. Rev. Lett., 82, 3440-3443, 1999.

Califano, F. and Lontano, M.: Vlasov simulations of strongly nonlinear electrostatic oscillations in a one-dimensional electron-ion plasma, Phys. Rev. E, 58, 6503-6511, 1998.

Cattel, C., Crumley, J., Dombeck, J., Wygant, J., and Mozer, F. S.: Polar observations of solitary waves at the Earth's magnetopause, Geophys. Res. Lett., 29, doi:10.1029/2001GL014046, 2002.

Dombeck, J., Cattell, C., Crumley, J., Peterson, W. K., Collin, H. L., and Kletzing, C.: Observed trends in auroral zone ion mode solitary wave structure characteristics using data from Polar, J. Geophys. Res., 106, 19013-19 021, 2001.

Daldorff, L. K. S., Guio, P., Børve, S., Pécseli, H. L., and Trulsen, J.: Ion phase space vortices in 3 spatial dimensions, Europhys. Lett., 54, 161-167, 2001.

Drake, J. F., Swisdak, M., Cattell, C., Shay, M. A., Rogers, B. N., Zeiler, A.: Formation of electron holes and particle energization during magnetic reconnection, Science, 299, 873-877, 2003.

Eliasson, B.: Outflow boundary conditions for the one-dimensional Vlasov-Poisson system, J. Sci. Comput., 16, 1-28, 2001.

Eliasson, B. and Shukla, P. K.: Production of nonisothermal electrons and Langmuir waves because of colliding ion holes and trapping of plasmons in an ion hole, Phys. Rev. Lett., 92, 95006/1-4, 2004a.

Eliasson, B., and Shukla, P. K.: Dynamics of electron holes in an electron-oxygen-ion plasma, Phys. Rev. Lett., 93, 45001/14, 2004b.

Ergun, R. E., Carlson, C. W., McFadden, J. P., Mozer, F. S., Muschietti, L., Roth, I., and Strangeway, R. J.: Debye-scale plasma structures associated with magnetic-field-aligned electric fields, Phys. Rev. Lett., 81, 826-829, 1998a.

Ergun, R. E., Carlson, C. W., McFadden, J. P., Mozer, F. S., Delory, G. T., Peria, W., Chaston, C. C., Temerin, M., Roth, I., Muchetti, L., Elphik, R., Strangeway, R., Pfaff, R., Cattell, C. A., Klumpar, D., Shelly, E., Peterson, W., Moebius, E., and Kistler, L.: FAST satellite observations of large-amplitude solitary structures, Geophys. Res. Lett., 25, 2041-2044, 1998 b.
Ergun, R. E., Su, Y.-J., Andersson, L.,Carlson, C. W., McFadden, J. P., Mozer, F. S., Newman, D. L., Goldman, M. V., and Strangeway, R. J.: Direct observation of localized parallel electric fields in a space plasma, Phys. Rev. Lett., 87, 45003/1-4, 2001.

Esirkepov, T. Zh., Kamenets, F. F., Bulanov, S. V., and Naumova, N. M.: Low-frequency relativistic electromagnetic solitons in collisionless plasmas, JETP Lett., 68, 36-41, 1998.

Farina, D. and Bulanov, S. V.: Relativistic electromagnetic solitons in the electron-ion plasma, Phys. Rev. Lett., 86, 5289-5292, 2001.

Franck, C., Klinger, T., Piel, A., and Schamel, H.: Dynamics of periodic ion holes in a forced beam-plasma experiment, Phys. Plasmas, 8, 4271-4274, 2001.

Goldman, M. V.: Strong turbulence of plasma waves, Rev. Mod. Phys., 56, 709-735, 1984.

Gradshteyn, I. S. and Ryzhik, I. M.: Table of Integrals Series and Products, 4th ed., Academic, New York, 1965.

de Groot, S. R., van Leeuwen, W. A., and Weert, C. G.: Relativistic Kinetic Theory, Principles and Applications, North-Holland, Amsterdam, 1980.

Guio, P., Børve, S., Daldorff, L. K. S., Lynov, J. P., Michelsen, P., Pécseli, H. L., Rasmussen, J. J., Saeki, K., and Trulsen, J.: Phase space vortices in collisionless plasmas, Nonlin. Proc. Geophys., 10, 75-86, 2003,

SRef-ID: 1607-7946/npg/2003-10-75.

Guio, P. and Pécseli, H. L.: Phase space structures generated by an absorbing obstacle in a streaming plasma, Geophys. Res. Lett., 31, L03806/1-4, doi:10.1029/2003GL018461, 2004.

Hasegawa, A: Stimulated modulational instabilities of plasma waves, Phys. Rev. A, 1, 1746-1750, 1970.

Holloway, J. P. and Dorning, J. J.: Undamped plasma waves, Phys. Rev. A, 44, 3856-3868, 1991.

Hoshino, M.: Coupling across many scales, Science, 299, 834-835, 2003.

Ikezi, H., Chang, R. P. H., and Stern, R. A.: Nonlinear evolution of the electron-beam-plasma instability, Phys. Rev. Lett., 36, 1047$1051,1976$.

Intrator, T., Chan, C., Hershkowitz, N., and Diebold, D.: Nonlinear self-contraction of electron waves, Phys. Rev. Lett., 53, 1233 $1235,1984$.

Isaacson, E. and Keller, H. B.: Analysis of Numerical Methods, Dover, New York, 1994.

Karpman, V. I.: High frequency electromagnetic field in plasma with negative dielectric constant, Plasma Phys., 13, 477-490, 1971.

Karpman, V. I.; On the dynamics of sonic-Langmuir solitons, Phys. Scr., 11, 263-265, 1975a.

Karpman, V. I.: Nonlinear Waves in Dispersive Media, Pergamon, Oxford, 1975b.

Kaw, P. K., Sen, A., and Katsouleas, T.: Nonlinear 1D laser pulse solitons in a plasma, Phys. Rev. Lett., 68, 3172-3175, 1992.

Krasovsky, V. L., Matsumoto, H., and Omura, Y.: Interaction dynamics of electrostatic solitary waves, Nonlin. Proc. Geophys., 6, 205-209, 1999 ,

SRef-ID: 1607-7946/npg/1999-6-205.

Lancellotti, C. and Dorning, J. J.: Nonlinear Landau damping in a collisionless plasma, Phys. Rev. Lett., 80, 5236, 1998.

Lele, S. K.: Compact finite difference schemes with spectral-like resolution, J. Comput. Phys., 103, 16-42, 1992.

Manfredi, G.: Long-time behavior of nonlinear Landau damping, Phys. Rev. Lett., 79, 2815-2818, 1997.

Matsumoto, H. Kojima, H., Miyatake, T., Omura, Y., Okada, M., 
Nagano, I., and Tsutsui, M.: Electrostatic Solitary Waves (ESW) in the magnetotail: BEN wave forms observed by Geotail, Geophys. Res. Lett., 21, 2915-2918, 1994; Relation between electrostatic solitary waves and hot plasma flow in the plasma sheet boundary layer: Geotail observations, Geophys. Res. Lett., 21, 2919-2922, 1994.

McFadden, J. P., Carlson, C. W., Ergun, R. E., Mozer, F. S., Muschietti, L., Roth, I., and Moebius, E.: FAST observations of ion solitary waves, J. Geophys. Res., 108, 8018, doi:10.1029/2002JA009485, 2003.

Medvedev, M. V., Dimond, P. H., Rosenbluth, M. N., and Shevchenko, V. I.: Asymptotic theory of nonlinear Landau damping and particle trapping in waves of finite amplitude, Phys. Rev. Lett., 81, 5824-5826, 1998.

Mendonça, J. T.: Theory of Photon Acceleration, Institute of Physics, Bristol, 2001.

Mokhov, Yu V. and Chukbar, K. V.: Langmuir solitons of the Bernstein-Greene-Kruskal type, Fiz. Plazmy, 10, 206-208, 1984 (Sov. J. Plasma Phys., 10, 122-123, 1984).

Montgomery, D. S., Focia, R. J., Rose, H. A., Russell, D. A., Cobble, J. A., Fernández, J. C., and Johnson, R. P.: Observation of stimulated electron-acoustic-wave scattering, Phys. Rev. Lett., $87,155001 / 1-4,2001$

Nakamura, Y., Bailung, H., and Shukla, P. K.: Observation of ionacoustic shocks in a dusty plasma, Phys. Rev. Lett., 83, 1602$1605,1999$.

Naumova, N. M., Bulanov, S. V., Esirkepov, T. Zh., Farina, D., Nishihara, K., Pegoraro, F., Ruhl, H., and Sakharov, A. S.: Formation of electromagnetic postsolitons in plasmas, Phys. Rev. Lett., 87, 185004/1-4, 2001.

Newman, D. L., Goldman, M. V., Spector, M., and Perez, F.: Dynamics and instability of electron phase-space tubes, Phys. Rev. Lett., 86, 1239-1242, 2001.

Pécseli, H. L., Trulsen, J., and Armstrong, R. J.: Experimental observation of ion phase-space vortices, Phys. Lett., 81A, 386-390, 1981.

Pécseli, H. L., Trulsen, J., and Armstrong, R. J.: Formation of ion phase-space vortices, Phys. Scripta, 29, 241-253, 1984.

Pécseli, H. L.: Ion phase-space vortices and their relation to small amplitude double-layers, Laser Part. Beams, 5, 211-217, 1987.

Petraconi, G. and Maciel, H. S.: Formation of electrostatic doublelayers and electron-holes in a low pressure mercury plasma column, J. Phys. D: Appl. Phys., 36, 2798-2805, 2003.

Pickett, J. S., Menietti, J. D., Gurnett, D. A., Tsurutani, B., Kintner, P. M., Klatt, E., and Balogh, A.: Solitary potential structures observed in the magnetosheath by the Cluster spacecraft, Nonlin. Proc. Geophys., 10, 3-11, 2003,

SRef-ID: 1607-7946/npg/2003-10-3.

Pickett, J. S., Kahler, S. W., Chen, L.-J., Huff, R. L., Santolik, O., Khotyaintsev, Y., Décréau, P. M. E., Winningham, D., Frahm, R., Goldstein, M. L., Lakhina, G. S., Tsurutani, B. T., Lavraud, B., Gurnett, D. A., André, M., Fazakerley, A., Balogh, A., and Rème, H.: Solitary waves observed in the auroral zone: the Cluster multi-spacecraft perspective, Nonlin. Proc. Geophys., 11, 183-196, 2004,

\section{SRef-ID: 1607-7946/npg/2004-11-183.}

Piran, T.: Gamma-ray bursts and the fireball model, Phys. Rep., 314, 575-667, 1999.
Rao, N. N., and Varma, R. K.: A theory for Langmuir solitons, J. Plasma Phys., 27, 95-120, 1982.

Rudakov, L. I.: Deceleration of electron beams in a plasma with a high level of Langmuir turbulence, Dokl. Akad. Nauk SSSR, 207, 821-823, 1972 (Sov. Phys. Dokl., 17, 1116, 1973).

Saeki, K. and Genma, H.: Electron-hole disruption due to ion motion and formation of coupled hole and ion-acoustic soliton in a plasma, Phys. Rev. Lett., 80, 1224-1227, 1998.

Saeki, K., Michelsen, P., Pécseli, H. L., and Rasmussen, J. J.: Formation and coalescence of electron solitary holes, Phys. Rev. Lett., 42, 501-504, 1979.

Sagdeev, R. Z.: in Reviews of Plasma Physics, edited by M. A. Leontovich, Consultants Bureau, New York, 4, 23m, 1966.

Schamel, H.: Stationary solutions of the electrostatic Vlasov equation, Plasma Phys., 13, 491-505, 1971.

Schamel, H.: Stationary solitary, snoidal and sinusoidal ion acoustic waves, Plasma Phys., 14, 905-924, 1972.

Schamel, H., Yu, M. Y., and Shukla, P. K.: Finite amplitude envelope solitons, Phys. Fluids, 20, 1286-1288, 1977.

Schamel, H.: Theory of electron holes, Phys. Scr., 20, 336-342, 1979.

Schamel, H.: Stability of electron vortex structures in phase space, Phys. Rev. Lett., 48, 481-483, 1982.

Schamel, H.: Electron holes, ion holes and double layers, Phys. Rep., 140, 161-191, 1986.

Schamel, H. and Maslov, V.: Langmuir contraction caused by electron holes, Phys. Scr., T82, 122-124, 1999.

Schamel, H.: Hole equilibria in Vlasov-Poisson systems: A challenge to wave theories of ideal plasmas, Phys. Plasmas, 7, 48314844, 2000.

Shapiro, V. D. and Shevchenko, V. I.: in Handbook of Plasma Physics, Vol. II, p. 8, North Holland, Amsterdam, 1984.

Shukla, P. K., Rao, N. N., Yu, M. Y., and Tsintsadze, N. L.: Relativistic nonlinear effects in plasmas, Phys. Rep., 138, 1-49, 1986.

Shukla, P. K. and Eliasson, B.: Trapping of plasmons in ion holes, JETP Lett., 77, 647-652, 2003 (Zh. Eksp. Teor. Fiz., 77, 778783, 2003)

Varma, R. K. and Rao, N. N.: Theory of sonic-Langmuir solitons, Phys. Lett. A, 79, 311-314, 1980.

Vetoulis, G. and Oppenheim, M.: Electrostatic mode excitation in electron holes due to wave bounce resonances, Phys. Rev. Lett., 86, 1235-1238, 2001.

Wang, J. G., Newman, D. L., and Goldman, M. V.: Vlasov simulations of electron heating by Langmuir turbulence near the critical altitude in the radiation-modified ionosphere, J. Atm. Solar-Terr. Phys., 59, 2461-2474, 1997.

Wong, A. Y. and Quon, B. H.: Spatial collapse of beam-driven plasma waves, Phys. Rev. Lett., 34, 1499-1502, 1975.

Wong, H. C., Stenzel, R., and Wong, A. Y.: Development of "cavitons" and trapping of rf field, Phys. Rev. Lett., 33, 886-889, 1974.

Yan'kov, V. V.: Two types of Langmuir solitons, Pis'ma Zh. Eksp. Teor. Fiz., 29, 179-180, 1979 (JETP Lett., 29, 160-161, 1979).

Zakharov, V. E.: Collapse of Langmuir waves, Zh. Eksp. Teor. Fiz., 62, 1745-1751, 1972 (Sov. Phys. JETP, 35, 908-914, 1972). 